\title{
Synergistic radar and radiometer retrievals of ice hydrometeors
}

\author{
Simon Pfreundschuh ${ }^{1}$, Patrick Eriksson ${ }^{1}$, Stefan A. Buehler ${ }^{2}$, Manfred Brath $^{2}$, David Duncan ${ }^{1, a}$, Richard Larsson ${ }^{3}$, \\ and Robin Ekelund ${ }^{1}$ \\ ${ }^{1}$ Department of Space, Earth and Environment, Chalmers University of Technology, 41296 Gothenburg, Sweden \\ ${ }^{2}$ Meteorologisches Institut, Fachbereich Geowissenschaften, Centrum für Erdsystem und Nachhaltigkeitsforschung (CEN), \\ Universität Hamburg, Bundesstraße 55, 20146 Hamburg, Germany \\ ${ }^{3}$ Max Planck Institute for Solar System Research, Justus-von-Liebig-Weg 3, 37077 Göttingen, Germany \\ ${ }^{a}$ now at: European Centre for Medium-Range Weather Forecasts, Shinfield Park, Reading RG2 9AX, UK
}

Correspondence: Simon Pfreundschuh (simon.pfreundschuh@chalmers.se)

Received: 1 October 2019 - Discussion started: 21 October 2019

Revised: 11 June 2020 - Accepted: 3 July 2020 - Published: 12 August 2020

\begin{abstract}
Remote sensing observations at sub-millimeter wavelengths provide higher sensitivity to small hydrometeors and low water content than observations at millimeter wavelengths, which are traditionally used to observe clouds and precipitation. They are employed increasingly in field campaigns to study cloud microphysics and will be integrated into the global meteorological observing system to measure the global distribution of ice in the atmosphere with the launch of the Ice Cloud Imager (ICI) radiometer on board the second generation of European operational meteorological satellites (Metop-SG). Observations at these novel wavelengths provide valuable information not only on their own but also in combination with complementary observations at other wavelengths. This study investigates the potential of combining passive sub-millimeter radiometer observations with a hypothetical W-band cloud radar for the retrieval of frozen hydrometeors. An idealized cloud model is used to investigate the information content of the combined observations and establish their capacity to constrain the microphysical properties of ice hydrometeors. A synergistic retrieval algorithm for airborne observations is proposed and applied to simulated observations from a cloud-resolving model. Results from the synergistic retrieval are compared to equivalent radar- and passive-only implementations in order to assess the benefits of the synergistic sensor configuration. The impact of the assumed ice particle shape on the retrieval results is assessed for all retrieval implementations. We find that the combined observations better constrain the microphysical properties of ice hydrometeors, which reduces uncertainties in retrieved ice water content and particle number
\end{abstract}

concentrations for suitable choices of the ice particle model. Analysis of the retrieval information content shows that, although the radar contributes the largest part of the information in the combined retrieval, the radiometer observations provide complementary information over a wide range of atmospheric states. Furthermore, the combined observations yield slightly improved retrievals of liquid cloud water in mixed-phase clouds, pointing towards another potential application of combined radar-radiometer observations.

\section{Introduction}

Ice hydrometeors play an important role for both weather and climate. They influence the Earth's energy budget through their interaction with incoming and outgoing radiation, constitute a part of the global hydrological cycle, and are coupled to the dynamics of the atmosphere in multiple ways (Bony et al., 2015). Because of this, observations of ice clouds are required for understanding the role of clouds in a changing climate (Boucher et al., 2013), to provide information on the dynamical state of the atmosphere in numerical weather prediction (NWP) models (Geer et al., 2017) and to validate climate models (Waliser et al., 2009). Despite this, today's global observing system cannot provide accurate information on the global distribution of ice in the atmosphere (Eliasson et al., 2011; Duncan and Eriksson, 2018). A major difficulty of measuring atmospheric ice using remote sensing lies in the large variability in ice particle sizes, concentrations 
and shapes, which can only be partially resolved by available spaceborne sensors.

Current operational observation systems used to study clouds can be divided into two groups by virtue of their observing frequency and corresponding capabilities and limitations. Microwave sensors employ comparably long wavelengths ranging down to about $1 \mathrm{~mm}$. Since these wavelengths are large compared to the typical sizes of ice particles in a cloud, microwave sensors are most sensitive to the largest particles and do not provide any sensitivity to the small particles in the cloud. Optical and infrared sensors use radiation with wavelengths from around $15 \mu \mathrm{m}$ down to several hundred nanometers. These relatively short wavelengths also make them sensitive to the small ice particles in the cloud. The comparably low sensitivity of microwave sensors to small ice particles allows them to sense the larger, potentially precipitating, particles typically located at the center and base of a cloud, which cannot be sensed at infrared and optical wave lengths due to saturation of the signal.

Active sensors have the advantage of providing high vertical resolution and generally higher sensitivity than their passive counterparts. This, however, typically comes at the expense of lower spectral and spatial coverage of the observations.

The most accurate current information on the global distribution of ice water content (IWC) is provided by the CloudSat radar. A main strength of these observations is their vertical resolution, on the order of $500 \mathrm{~m}$. However, the radar lacks scanning capability, and the swath width is just $1.5 \mathrm{~km}$ wide, to be contrasted with the swath width of passive imagers, which is on the order of $1000 \mathrm{~km}$. CloudSat performs a single-frequency measurement, which limits the information per range bin to 1 degree of freedom. Retrieving bulk properties like water content or particle number densities thus requires making a priori assumptions that constrain cloud microphysical properties such as particle size, concentration and shape.

A way to overcome the limitations of single-frequency radars is to combine them with observations from passive sensors, which typically provide measurements at multiple frequencies and a significantly wider swath. Two types of synergies can be distinguished for such an observation scenario: a local synergy, which consists of using the colocated radar and radiometer observations to obtain more accurate hydrometeor retrievals, and a non-local synergy, which uses the vertically well-resolved results from the radar-only or combined observations to support passive-only retrievals across the wide swath of the passive sensor, for example by providing realistic a priori constraints. Prominent examples of satellite missions that exploit both these synergies are the Tropical Rainfall Measuring Mission (TRMM; Kummerow et al., 1998; Grecu et al., 2004; Munchak and Kummerow, 2011) and the Global Precipitation Measurement (GPM) mission (Hou et al., 2014; Grecu et al., 2016; Kummerow et al., 2015). Since the principal target of these mis- sions is the retrieval of precipitation, they make use of comparably low microwave frequencies and hence provide only little sensitivity to non-precipitating hydrometeors (Greenwald and Christopher, 2002).

With the upcoming launch of the Ice Cloud Imager (ICI), a new passive microwave sensor will become operational that is dedicated to observing ice hydrometeors from space. ICI will extend the range of currently available microwave frequencies with channels at 243, 325, 448 and $664 \mathrm{GHz}$ (Eriksson et al., 2020). This will narrow the size-sensitivity gap between the infrared and traditional microwave sensors by extending the smallest currently available microwave wavelength from $1.6 \mathrm{~mm}$ at $183 \mathrm{GHz}$ down to the sub-millimeter domain $(0.45 \mathrm{~mm}$ at $664 \mathrm{GHz})$ and significantly improve the size sensitivity of spaceborne microwave observations of clouds. Together with ICI, the newly developed Microwave Imager (MWI) will be flown on the satellites of the MetopSG program. MWI will complement ICI's observations with measurements at traditional millimeter wavelengths as well as a spectral band around the $118 \mathrm{GHz}$ oxygen line. The observations of MWI, which cover the frequency range from 19 up to $183 \mathrm{GHz}$, will provide additional sensitivity to liquid and frozen precipitation as well as water vapor.

A number of studies have investigated the potential of ICI for studying ice in the atmosphere. The information content and retrieval performance of radiometer observations alone have been studied in detail for column-integrated ice water content (Jiménez et al., 2007; Wang et al., 2017; Brath et al., 2018; Eriksson et al., 2020) as well as for the vertical distribution of ice in the atmosphere (Birman et al., 2017; Grützun et al., 2018; Aires et al., 2019). Although not directly related to ICI, the combination of millimeter and sub-millimeter radiometer observations with active observations from a cloud radar has been investigated by Evans et al. (2005) and Jiang et al. (2019).

In this study, we are interested in the local synergies of colocated MWI and ICI-type radiometer observations combined with observations from a W-band radar. In particular, we aim to answer the question of what additional information can be gained from combined observations compared to observations from a radar or MWI and ICI alone. For this, a combined, variational retrieval is developed and applied to simulated observations of scenes from a cloud-resolving model (CRM). An airborne viewing geometry is assumed for the simulations with all sensors pointing at nadir and closeto-overlapping antenna beams. Our work extends the previous work by Evans et al. (2005) and Jiang et al. (2019) by comparing the performance of the combined retrieval to that of equivalent radar- and passive-only retrievals, which allows us to quantify the value added by the synergistic observations. In addition to that, the impact of the assumed scattering properties of ice hydrometeors on the retrieval is investigated.

This study consists of two principal parts. In the first part, simulated observations from a simplified cloud model are 


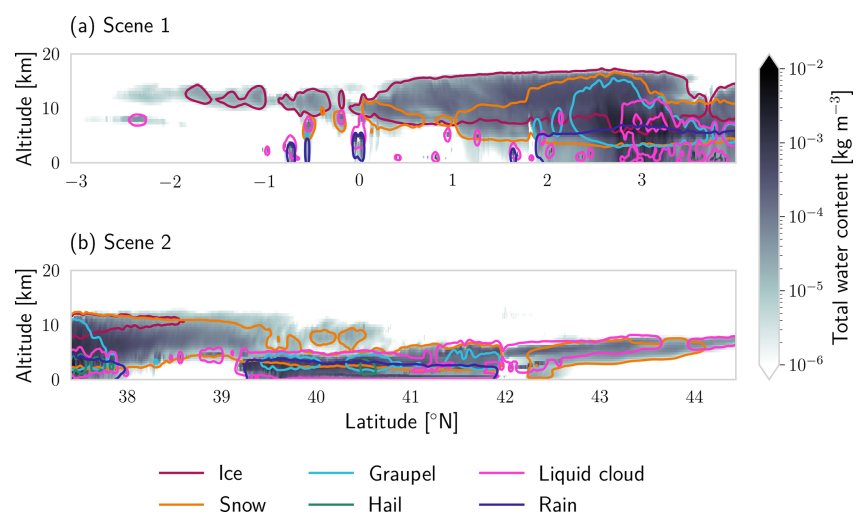

Figure 1. The distribution of total water content including all hydrometeor classes in the two cloud scenes used to test the retrieval. Colored lines show the $10^{-5} \mathrm{~kg} \mathrm{~m}^{-3}$ contour of the water content of each hydrometeor class.

used to perform a preliminary study of the complementary information content of radar and passive radiometer observations. In the second part, the developed synergistic retrieval algorithm is applied to simulated observations from a CRM to investigate the performance benefits of the combined observations compared to radar- and passive-only configurations. Following this introduction, Sect. 2 introduces the test data, sensor configuration and the developed retrieval algorithm on which the study is based. This is followed by the experimental results on the information content of the combined observations and the simulated retrieval results in Sect. 3. The article closes with a discussion of the results in Sect. 4 and conclusions in Sect. 5.

\section{Methods and data}

\subsection{Reference cloud scenes}

The cloud scenes which are used for the testing of the retrieval were produced by Environment and Climate Change Canada using a high-resolution NWP configuration of the Global Environmental Multiscale (GEM) Model (Côté et al., 1998). Two test scenes with a horizontal resolution of $1 \mathrm{~km}$ and an extent of $800 \mathrm{~km}$ were selected. The vertical resolution of the model scenes varies between 250 and $500 \mathrm{~m}$ below an altitude of $18 \mathrm{~km}$ and decreases steadily above that. The scenes, displayed in Fig. 1, were chosen with the aim of covering a large range of cloud structures and compositions so as to ensure a broad assessment of the retrieval. The first test scene, shown in panel (a), is located in the tropical Pacific and contains a mesoscale convective system in the northern half of the scene and its anvil, which extends into the southern half. The second scene, shown in panel (b), is located in the North Atlantic and contains an ice cloud in the southern part and a low-level, mixed-phase cloud in the northern part.
The GEM model uses a two-moment scheme with six classes of hydrometeors to represent clouds and precipitation (Milbrandt and Yau, 2005): two classes of liquid hydrometeors (rain and liquid cloud) and four of frozen hydrometeors (cloud ice, snow, hail and graupel). The particle size distribution (PSD) of each hydrometeor class is described by a three-parameter gamma distribution. The prognostic parameters of the model are the slope and intercept parameters of the PSD, which are derived from the predicted mixing ratios and number concentrations. The third parameter, which defines the shape of the PSD, is set to a fixed, species-specific value. For each hydrometeor species a specific mass-size relationship is assumed.

Examples of particle size distributions of frozen hydrometeors are displayed in Fig. 2. The assumed particle size distributions across different ice species vary mostly in their scaling with respect to size and concentration, whereas the shape shows less variability. An important characteristic can be identified here that will help the reader to better understand the retrieval results presented later: cloud ice is characterized by high particle number concentrations and small particle sizes, whereas snow has lower number concentrations and larger particles.

In order to simulate observations from the GEM model scenes, the hydrometeor classes of its microphysics scheme must be associated with particle shapes to define their radiometric properties. The ARTS single-scattering database, described in more detail below, contains particle models which were designed to be consistent with the mass-size relationships assumed in the GEM model. The particle shapes used to represent the GEM model's different hydrometeor types are listed together with their properties in Table 1.

\subsection{Simulated cloud observations}

An airborne sensor configuration is simulated to test the retrieval. The beams of all three sensors are assumed to point at nadir and to be perfectly coincident pencil beams. Multiple scattering effects in the radar observations as well as the effects of particle orientation are neglected. Although these assumptions may be justified for an airborne configuration, this will not be the case for spaceborne observations from ICI and MWI. Moreover, the incidence angles of the beams of ICI and MWI will be around $53^{\circ}$ at the Earth's surface. This further complicates the radiative transfer modeling since it requires handling a more complex colocation geometry for the nadir-pointing radar and the passive instruments. At offnadir viewing angles, polarization also needs to be taken into account, the effects of which can be several kelvin at the typical viewing angles of microwave imagers (Xie et al., 2015).

\subsubsection{Sensor configuration}

The sensor configuration assumed for the simulated observations includes the 11 highest-frequency channels of the 
(a) Cloud ice

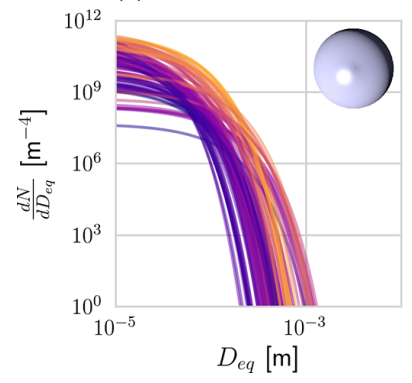

(b) Snow

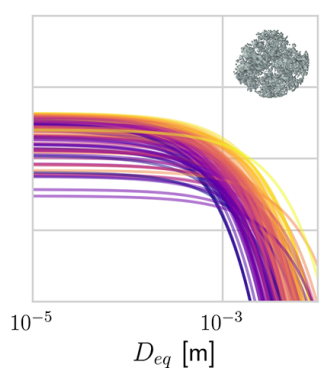

(c) Hail

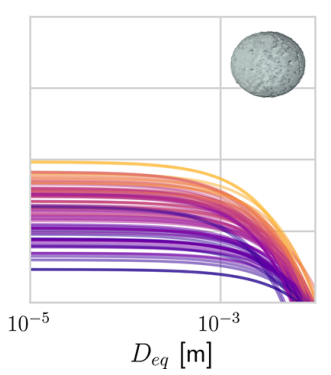

(d) Graupel

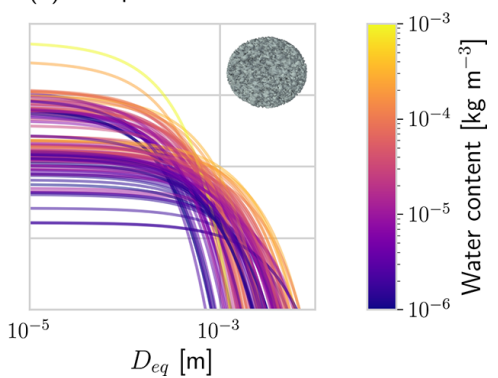

Figure 2. Realizations of particle size distributions from the test scenes used in this study. The particle number concentration is plotted with respect to the volume-equivalent diameter $D_{\text {eq }}$. Shown are the PSDs corresponding to 100 randomly chosen grid points with a water content higher than $10^{-6} \mathrm{~kg} \mathrm{~m}^{-3}$. Line color encodes the corresponding water content. Inlets display visualizations of the particle shape assumed for each hydrometeor species.

Table 1. Particle shapes used to represent the hydrometeor species of the GEM model scenes. The mass-size relationship is given in terms of the parameters of a fitted power law of the form $m=\alpha \cdot D_{\max }^{\beta}$, with $D_{\max }$ the maximum diameter in meters and $m$ in kilograms.

\begin{tabular}{llrr|rr}
\hline $\begin{array}{l}\text { GEM hydrometeor } \begin{array}{l}\text { Alass } \\
\text { classociated particle } \\
\text { shape }\end{array} \\
\text { Name (ID) }\end{array}$ & \begin{tabular}{r} 
Size range \\
\cline { 3 - 6 }
\end{tabular} & $\begin{array}{r}D_{\text {eq, min }} \\
(\mu \mathrm{m})\end{array}$ & $\begin{array}{r}D_{\text {eq, } \max }(\mu \mathrm{m}) \\
\text { relationship }\end{array}$ & $\alpha$ & $\beta$ \\
\hline Liquid cloud & LiquidSphere (25) & 1 & $5 \times 10^{4}$ & 480 & 3 \\
Rain & LiquidSphere (25) & 1 & $5 \times 10^{4}$ & 480 & 3 \\
Ice cloud & GEM Cloud Ice (31) & 10 & $3 \times 10^{3}$ & 440 & 3 \\
Snow & GEM Snow (32) & 94 & $5 \times 10^{3}$ & 24 & 2.86 \\
Graupel & GEM Graupel (33) & 94 & $5 \times 10^{3}$ & 170 & 2.96 \\
Hail & GEM Hail (34) & 94 & $5 \times 10^{3}$ & 540 & 3.02 \\
\hline
\end{tabular}

MWI radiometer and all ICI channels. For the radar, a nadirpointing, W-band cloud radar with similar characteristics as the CloudSat Cloud Profiling Radar (CPR; Stephens et al., 2002; Tanelli et al., 2008) is assumed.

Observations from the ICI radiometer are simulated by performing a single, non-polarized radiative transfer simulation located at the centers of the pass bands of each doublesideband channel and averaging the resulting brightness temperatures. For channels with multiple polarizations, only a single simulation is performed. To compensate for this, the noise of the corresponding channel is reduced by a factor of $\sqrt{2}$. The simulated ICI channels and assumed noise levels are presented in Table 2.

Observations from the MWI radiometer are simulated in a similar manner to those of ICI except that for MWI only channels with frequencies larger than or equal to $89 \mathrm{GHz}$ are used. The reason for this is that the footprints of the channels with frequencies lower than $89 \mathrm{GHz}$ will have a full width at half maximum of $50 \mathrm{~km}$ compared to only $10 \mathrm{~km}$ for the MWI's higher-frequency channels and $16 \mathrm{~km}$ for ICI's channels. For a spaceborne configuration, these channels were deemed unlikely to be beneficial for a synergistic retrieval due to the very small overlap of the footprints of these chan- nels with that of the radar. The included MWI channels are listed in Table 2.

The frequency of the cloud radar is chosen to be $94 \mathrm{GHz}$, similar to the CloudSat CPR. The vertical resolution of the nadir-pointing radar observations is assumed to be $500 \mathrm{~m}$, ranging from 0.5 to $20 \mathrm{~km}$ in altitude. The minimum sensitivity is set to be $-30 \mathrm{dBZ}$, and the noise at each range gate is modeled to be independent with standard deviation $0.5 \mathrm{~dB}$.

\subsubsection{Radiative transfer simulations}

All simulations presented in this study were performed using Version 2.3.1279 of the Atmospheric Radiative Transfer Simulator (ARTS; Buehler et al., 2018). Radar reflectivities are computed using ARTS' built-in single-scattering radar solver, which provides analytic Jacobians. For the simulation of passive radiances, a hybrid solver is used which combines the DISORT (Stamnes et al., 2000) scattering solver with the ARTS standard scheme for pencil beam radiative transfer. The hybrid solver has been added to ARTS specifically for this study and provides approximate, analytical Jacobians, which are required for variational retrievals of hydrometeors. All simulations are performed assuming an 
Table 2. Channels of the MWI and ICI radiometers used in the retrieval.

\begin{tabular}{lrr|lrr}
\hline \multicolumn{2}{c}{ MWI } & \multicolumn{3}{c}{ ICI } \\
\hline Channel & Freq. (GHz) & Noise (K) & Channel & Freq. (GHz) & Noise (K) \\
\hline MWI-8 & 89 & 1.1 & ICI-1 & $183.31 \pm 7.0$ & 0.8 \\
MWI-9 & $118.75 \pm 3.2$ & 1.3 & ICI-2 & \pm 3.4 & 0.8 \\
MWI-10 & \pm 2.1 & 1.3 & ICI-3 & \pm 2.0 & 0.8 \\
MWI-11 & \pm 1.4 & 1.3 & ICI-4 & $243 \pm 2.5$ & $\frac{1}{\sqrt{2}} \cdot 0.7$ \\
MWI-12 & \pm 1.2 & 1.3 & ICI-5 & $325.15 \pm 9.5$ & 1.2 \\
MWI-13 & $165.5 \pm 0.75$ & 1.2 & ICI-6 & \pm 3.5 & 1.3 \\
MWI-14 & $183.31 \pm 7.0$ & 1.3 & ICI-7 & \pm 1.5 & 1.5 \\
MWI-15 & \pm 6.1 & 1.2 & ICI-8 & $448 \pm 7.2$ & 1.4 \\
MWI-16 & \pm 4.9 & 1.2 & ICI-9 & \pm 3.0 & 1.6 \\
MWI-17 & \pm 3.4 & 1.2 & ICI-10 & \pm 1.4 & 2.0 \\
MWI-18 & \pm 2.0 & 1.3 & ICI-11 & $664 \pm 4.2$ & $\frac{1}{\sqrt{2}} \cdot 1.6$ \\
\hline
\end{tabular}

ocean surface with emissivities calculated using the Tool to Estimate Sea-Surface Emissivity from Microwaves to subMillimeter waves (TESSEM; Prigent et al., 2017). Polarization is neglected in all simulations performed in this study. Gaseous absorption is modeled using the absorption models from Rosenkranz (1993) for $\mathrm{N}_{2}$ and $\mathrm{O}_{2}$ and from Rosenkranz (1998) for $\mathrm{H}_{2} \mathrm{O}$.

Single-scattering data for hydrometeors are taken from ARTS single-scattering database (ARTS SSDB; Eriksson et al., 2018). The database provides scattering data for a wide range of hydrometeor shapes including particles designed specifically to be consistent with assumptions of the GEM microphysics scheme. It also provides a number of predefined habit mixes, referred to as standard habits, which cover the full range of particle sizes relevant for microwave observations of ice hydrometeors.

\subsection{Retrieval algorithm}

A one-dimensional, variational cloud retrieval algorithm is proposed, which uses the optimal estimation method (OEM; Rodgers, 2000) to fit an atmospheric state to given observations. The quality of a retrieved state $\hat{\boldsymbol{x}}$ and corresponding simulated observations $\hat{\boldsymbol{y}}$ is assessed using the following diagnostic quantity:

$\chi_{y}^{2}=\Delta \boldsymbol{y}^{T} \mathbf{S}_{\mathrm{e}}^{-1} \Delta \boldsymbol{y}$.

Here, $\Delta \boldsymbol{y}=\boldsymbol{y}-\hat{\boldsymbol{y}}$ is the difference between the true and fitted observations, and $\mathbf{S}_{\mathrm{e}}$ is the covariance matrix describing the measurement errors. The quantity $\chi_{y}^{2}$ corresponds to the sum of squared errors in the fitted observations weighted by the precision of each channel or range bin. It should be noted that the quantity has no meaningful interpretation in terms of the $\chi^{2}$ statistic for the errors in the fitted observations since they will be neither independent (cf. chap. 12 in Rodgers, 2000) nor Gaussian due to the presence of forward model error. The value is therefore used here solely as a heuristic to quantify the goodness of the fit to the true observations.

\subsubsection{Measurement space}

The input for the synergistic retrieval is the combined observation vector $\boldsymbol{y}$ consisting of the concatenated singleinstrument observations from the cloud radar and the two radiometers. Measurement errors are assumed to be independent and Gaussian distributed with standard deviations according to the noise characteristics given in Sect. 2.2.1. For the single-instrument retrievals, the measurement vector consists only of observations from either the radar or the radiometers.

\subsubsection{State space}

The proposed retrieval solves for profiles of 2 degrees of freedom of the PSDs of frozen hydrometeors and rain along with profiles of relative humidity $(\mathrm{RH})$ and cloud liquid water content (CLWC). An illustration of the retrieved quantities and their respective retrieval grids for the combined and singleinstrument configurations of the retrieval are given in Fig. 3.

The PSDs of frozen hydrometeors and rain are represented using the normalized particle size distribution formalism proposed by Delanoë et al. (2005). The PSD of a hydrometeor species at a given altitude is modeled using a generalized gamma distribution function with four parameters. The massweighted mean diameter $D_{\mathrm{m}}$, which scales the PSD along the size dimension, and the normalized number density $N_{0}^{*}$, which scales the particle concentration, are the 2 retrieved degrees of freedom of the PSD. The other two parameters describe the shape of the normalized PSD. The same shape parameters as in version 3 of the DARDAR-CLOUD product (Cazenave et al., 2019) are chosen for frozen hydrometeors. For rain, they are chosen to match the shape used in the GEM model for rain drops. 


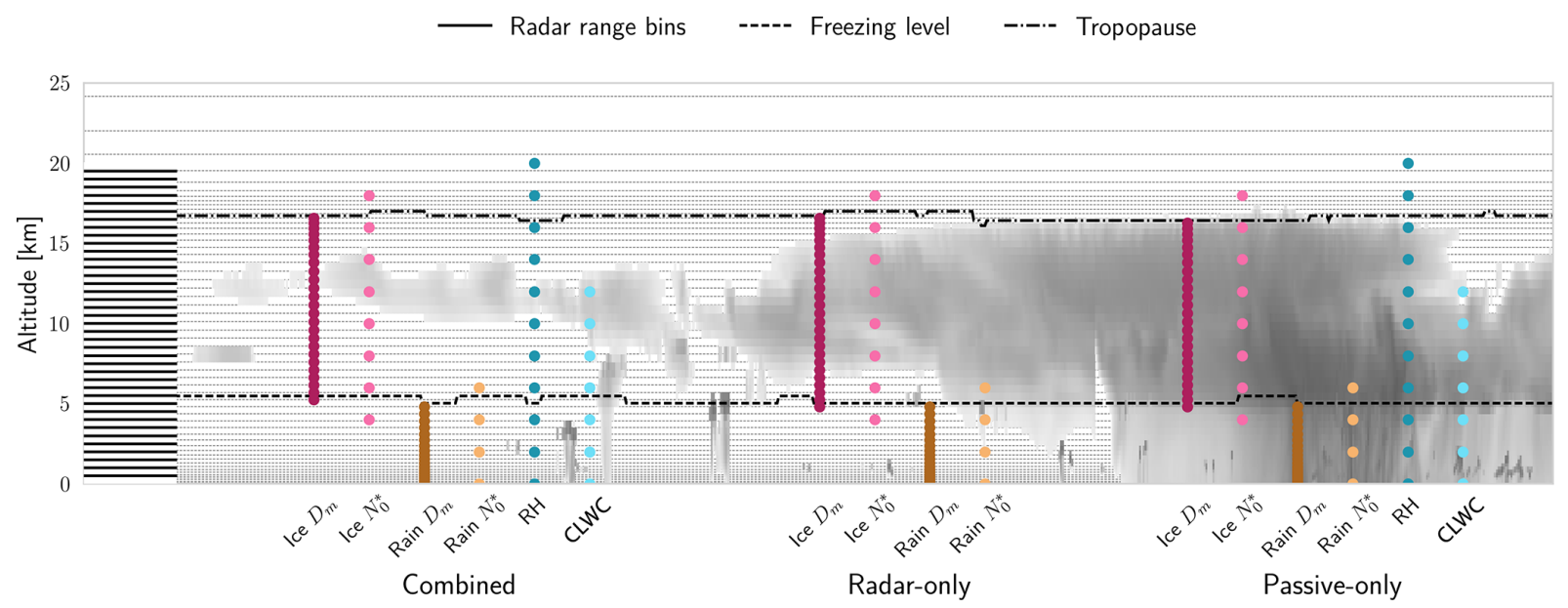

Figure 3. Illustration of retrieval quantities and their respective retrieval grids. Dotted grey lines in the background display the vertical grid of the GEM model. Solid black lines on the left side display the range bins of the radar observations. Filled markers represent the retrieval grids of each retrieval quantity for the combined, radar-only and passive-only configurations of the retrieval algorithm.

The temperature-dependent a priori profile for $N_{0}^{*}$ for frozen hydrometeors is determined using the relation from Delanoë et al. (2014)

$N_{0}^{*}=\exp (-0.076586 \cdot(T-273.15)+17.948)$,

where $T$ is in kelvin. The a priori profile of $D_{\mathrm{m}}$ for frozen hydrometeors is chosen so that the a priori IWC is equal to $10^{-6} \mathrm{~kg} \mathrm{~m}^{-3}$. For rain, a fixed value for $N_{0}^{*}$ of $10^{6} \mathrm{~m}^{-4}$ is assumed, and the a priori profile for $D_{\mathrm{m}}$ is determined similarly as for frozen hydrometeors.

Since the $N_{0}^{*}$ parameters vary over several orders of magnitude, they are retrieved in $\log _{10}$ space for both frozen hydrometeors and rain. The $D_{\mathrm{m}}$ parameters, in contrast, are retrieved in linear space. Alternative parametrizations using water content and $D_{\mathrm{m}}$ or the water content and $N_{0}^{*}$ have been tested, but no considerable effect on retrieval performance has been observed. As additional constraints, the retrieval of frozen hydrometeors is restricted to the region between the freezing level, here defined simply as the $273.15 \mathrm{~K}$ isotherm, and the approximate altitude of the tropopause. The altitude of the tropopause is approximated as the first grid point at which the lapse rate is negative and temperature below $220 \mathrm{~K}$. The retrieval of rain hydrometeors is restricted to below the freezing level. The retrieval of the $N_{0}^{*}$ parameters is further regularized by retrieving them at reduced vertical resolution of $2 \mathrm{~km}$. This was found necessary in order to keep the retrieval from getting stuck in spurious local minima. A similar approach is taken in the GPM combined precipitation retrievals (Grecu et al., 2016), where the PSD parameter scaling the particle concentration is also retrieved at reduced resolution.

Relative humidity is retrieved at a vertical resolution of $2 \mathrm{~km}$. However, the values are not retrieved directly, but instead an inverse hyperbolic tangent transformation is applied to the relative humidity profile as follows:

$x=\operatorname{arctanh}\left(\frac{2 \mathrm{RH}}{1.2}-1.0\right)$.

The transformation restricts the retrieved relative humidity values to the range between $0 \%$ and $120 \%$. The a priori profile for relative humidity is set to

$\mathrm{RH}(t)= \begin{cases}0.7, & 270 \mathrm{~K}<t \\ 0.7-0.01 \cdot(270-t), & 220<t \leq 270 \mathrm{~K} \\ 0.2, & t<220 \mathrm{~K} .\end{cases}$

CLWC is retrieved at a resolution of $2 \mathrm{~km}$ but is restricted to the region between the surface and the $230 \mathrm{~K}$ isotherm. In contrast to frozen hydrometeors and rain, the PSD of liquid cloud droplets is not explicitly resolved in the retrieval forward model. Instead, liquid cloud droplets are modeled as a purely absorbing quantity using the model by Liebe et al. (1993) for suspended liquid cloud droplets. Note that this is the case only for the retrieval. For the simulated observations, liquid cloud droplets are handled as any other hydrometeor species in the GEM model. CLWC is retrieved in $\log _{10}$ space, and the a priori profile is set to a fixed value of $10^{-6} \mathrm{~kg} \mathrm{~m}^{-3}$ in the permitted region of the atmosphere.

The a priori distributions of the six retrieval quantities $\left(N_{0}^{*}\right.$ and $D_{\mathrm{m}}$ for frozen and liquid hydrometeors, RH and CLWC) are assumed to be independent so that the overall a priori covariance matrix $\mathbf{S}_{\mathrm{a}}$ has block-diagonal structure. Within each block, vertical correlations between the values of a given retrieval quantity at different altitudes are assumed to be exponentially decaying. The covariance of the values of retrieval quantity $q$ at points $i$ and $j$ of the retrieval grid is computed as

$\left(\mathbf{S}_{\mathrm{a}, q}\right)_{i, j}=\sigma_{q, i} \sigma_{q, j} \cdot \exp \left(-\frac{d(i, j)}{l_{q}}\right)$, 
Table 3. A priori uncertainties $\sigma_{q}$ and correlation lengths $l_{q}$ used in the retrieval.

\begin{tabular}{ll|lr|lr}
\hline \multicolumn{2}{c|}{ Retrieval target } & \multicolumn{2}{c}{$\begin{array}{c}\text { Combined/ } \\
\text { radar-only }\end{array}$} & \multicolumn{2}{c}{ Passive-only } \\
\hline Name & Retrieved quantity & $\sigma_{q}$ & $l_{q}(\mathrm{~km})$ & $\sigma_{q}$ & $l_{q}(\mathrm{~km})$ \\
\hline Ice, $N_{0}^{*}$ & $\log _{10}\left(N_{0, \text { Ice }}^{*}\right)$ & 2 & 2 & 2 & 5 \\
Ice, $D_{\mathrm{m}}$ & $\operatorname{Ice}_{\mathrm{m}, \text { Ice }}$ & $300 \mu \mathrm{m}$ & 2 & $300 \mu \mathrm{m}$ & 5 \\
Rain, $N_{0}^{*}$ & $\log _{10}\left(\right.$ Rain $\left.N_{0}^{*}\right)$ & 2 & 2 & 2 & 5 \\
Rain, $D_{\mathrm{m}}$ & $D_{\mathrm{m}, \text { Rain }}$ & $300 \mu \mathrm{m}$ & 2 & $300 \mu \mathrm{m}$ & 5 \\
Relative humidity $(\mathrm{RH})$ & $\left.\operatorname{arctanh}^{2} \frac{2 \cdot \mathrm{RH}}{1.2}-1.0\right)$ & $0.5^{\mathrm{a}}$ & $2^{\mathrm{a}}$ & 0.5 & 2 \\
Cloud liquid water content (CLWC) & $\log _{10}(\mathrm{CLWC})$ & $1^{\mathrm{a}}$ & $2^{\mathrm{a}}$ & 1 & 2 \\
\hline
\end{tabular}

a Not retrieved in radar-only retrieval

where $\sigma_{q, i}$ is the a priori uncertainty assumed for retrieval quantity $q$ at grid point $i$; $d(i, j)$ is the vertical distance between the grid points, and $l_{q}$ the quantity-specific correlation length. The assumed a priori uncertainties and correlation lengths for the retrieval quantities are summarized in Table 3.

The radar-only version of the retrieval is similar to the combined version except that $\mathrm{RH}$ and CLWC are not retrieved. Instead, perfect knowledge of the true $\mathrm{RH}$ profile is assumed, while CLWC is neglected. In addition to a twomoment, radar-only retrieval, a one-moment version (M1), in which only the $D_{\mathrm{m}}$ parameter is retrieved has also been tested. However, results of this version will be shown only for the comparison of IWC retrieval errors. For the passiveonly retrieval, the retrieval quantities and grids are the same as for the combined retrieval. However, higher correlation lengths are assumed, which are shown in Table 3

\subsubsection{Representation of ice particle shape}

A major difficulty for cloud retrievals is that the observations may not provide sufficient information to distinguish different species of hydrometeors. Due to this ambiguity, frozen hydrometeors in the proposed retrieval algorithm are represented using only a single hydrometeor species. It is therefore necessary to find a suitable representation for frozen hydrometeors that can capture the variability in the four frozen hydrometeor species in the GEM model and ideally also that of real ice hydrometeors.

The four species of frozen hydrometeors in the GEM model have different characteristic particle concentrations, sizes and shapes (cf. Fig. 2). Since the retrieval can adapt 2 degrees of freedom of the PSD of frozen hydrometeors, it can represent the variations in particle number concentrations and particle sizes of the different hydrometeor species. By using a habit mix for the ice hydrometeor shape used in the retrieval, variations in particle shape that correlate with particle size, such as differences between pristine crystals and aggregates or rimed particles, can be represented in the retrieval.
Even with this configuration, the single hydrometeor species used in the retrieval is unlikely to be able to represent the variability present in the GEM model or the real world. To shed some light on the question of which particle shape should be assumed in the retrieval to minimize the resulting representation error, we chose a set of multiple particle shapes and habit mixes for which we investigated the impact of the particle choice on the retrieval results.

The selected particles are listed in Table 4. Three of them, GEM Cloud Ice, GEM Snow and GEM Graupel, correspond to the shapes present in the GEM model scenes. The GEM Snow and Graupel habits were mixed with crystal shapes to ensure that they cover sizes down to around $10 \mu \mathrm{m}$. In addition to this, two of the habit mixes distributed with the ARTS SSDB, the Large Plate Aggregate and Large Column Aggregate standard habits, are included in the selection to increase the range of scattering properties it covers.

Figure 4 provides an overview of the bulk mass backscattering and attenuation coefficients of the selected particles at the frequency of the cloud radar and three selected frequencies of the passive radiometers. Mass backscattering and attenuation coefficients are defined as the ratio of the corresponding backscattering or attenuation coefficient $\sigma$ and the bulk water content WC:

$Q=\frac{\sigma}{\mathrm{WC}}$.

For each particle shape and frequency, $Q$ has been computed for three different values of the $N_{0}^{*}$ parameter of the PSD. For a fixed bulk-mass, the value of the $N_{0}^{*}$ parameter of the PSD is related to the size of the bulk particles: for high $N_{0}^{*}$ values the number of large particles is decreased, while it is increased for low $N_{0}^{*}$ values. The variation in the mass backscattering and attenuation coefficients with mass show the nonlinear relationship between bulk mass and the particles' radiometric properties. For high values of $N_{0}^{*}$, which are typical for cloud ice, the radiometric properties of particle shapes differ only for large masses at the two highest frequencies considered. For low $N_{0}^{*}$ values, which are more typical for snow, the particles' properties differ considerably 
Table 4. Particle models used to represent ice hydrometeors used in the retrieval. The mass-size relationship is given in terms of the parameters of a fitted power law of the form $m=\alpha \cdot D_{\max }^{\beta}$, with $D_{\max }$ the maximum diameter in meters and $m$ in kilograms.

\begin{tabular}{llrr|rr}
\hline Name & Shapes used & Size range & $\begin{array}{c}\text { Mass-size } \\
\text { relationship }\end{array}$ \\
\cline { 2 - 6 } & Name (ID) & $\begin{array}{r}D_{\text {eq, }} \text { min } \\
(\mu \mathrm{m})\end{array}$ & $\begin{array}{r}D_{\text {eq, }} \\
(\mu \mathrm{m})\end{array}$ & $\alpha$ & $\beta$ \\
\hline GEM Cloud Ice & GEM Cloud Ice (31) & 10 & 3000 & 440 & 3 \\
\hline GEM Snow & 8-Column Aggregate (8) & 10 & 127 & 65 & 3 \\
& GEM Snow (32) & 107 & 5000 & 24 & 2.86 \\
\hline GEM Graupel & 8-Column Aggregate (8) & 10 & 179 & 65 & 3 \\
& GEM Graupel (33) & 107 & 5000 & 170 & 2.96 \\
\hline Large Plate Aggregate & Thick Plate (15) & 16 & 200 & 110 & 3 \\
& Large Plate Aggregate (33) & 160 & 3021 & 0.21 & 2.26 \\
\hline Large Column Aggregate & Block Column (12) & 10 & 200 & 210 & 3 \\
& Large Column Aggregate (18) & 160 & 3021 & 0.25 & 2.43 \\
\hline
\end{tabular}

at all masses and frequencies. At the two lowest frequencies, the Large Column Aggregate, the Large Plate Aggregate and GEM Snow are the least efficient in scattering or absorbing radiation, whereas GEM Graupel, GEM Hail and GEM Cloud Ice are more efficient. This behavior is also observed at the two higher frequencies, except for the lowest $N_{0}^{*}$ value for which a reversal of the ordering occurs as the bulk mass increases. The mass backscattering efficiency at $94 \mathrm{GHz}$ shows the greatest relative variability across different bulk water contents and $N_{0}^{*}$ values, spanning 6 orders of magnitude, while for the mass attenuation coefficients at the other frequencies, the variability spans at most 3 orders of magnitude.

\section{Results}

The first part of this section presents results from a numerical experiment which investigates the complementary information content of the active and passive microwave observations. Results of the combined and single-instrument retrievals applied to the reference cloud scenes are presented in the second part.

\subsection{Complementary information content}

A fundamental question regarding the benefit of combining two remote sensing observations in a retrieval is to what extent the observations contain non-redundant information. The degree of non-redundancy in the combined observations is what we refer to here as complementary information content. We are thus interested in the information that cannot be provided by either of the instruments alone. As an example, we do not consider the high vertical resolution achieved by combining passive with radar observations as complemen-
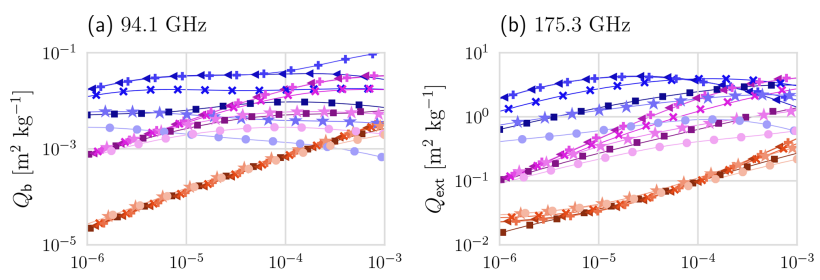

(c) $314.2 \mathrm{GHz}$

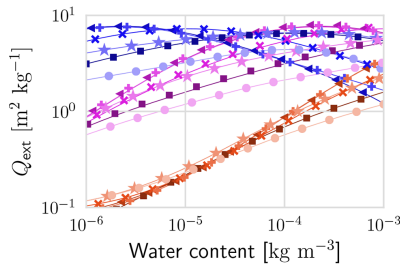

(d) $657.3 \mathrm{GHz}$

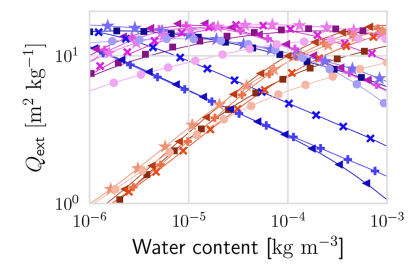

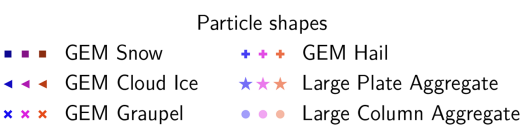

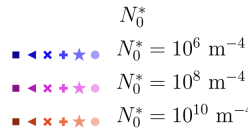

Figure 4. Bulk mass backscattering coefficient $Q_{\mathrm{b}}$ at (a) $94.1 \mathrm{GHz}$ and mass attenuation coefficients $Q_{\mathrm{e}}$ at frequencies (b) $175.3 \mathrm{GHz}$, (c) $314.2 \mathrm{GHz}$ and (d) $657.3 \mathrm{GHz}$ for the particle models used in the simulated observations and the retrieval. Different colors show the bulk properties for different values of the $N_{0}^{*}$ parameter of the PSD.

tary information since the same vertical resolution would be provided by radar-only observations.

In order to explore the complementary information content in the radar and radiometer observations, an idealized, homogeneous cloud layer with a thickness of $5 \mathrm{~km}$ centered at an altitude of $10 \mathrm{~km}$ in a tropical atmosphere is considered. The cloud is assumed to consist of a single species of frozen hydrometeors represented using the PSD parametrization, which is also used in the retrieval and described in 
Sect. 2.3.2. As the particle model, the 8-Column Aggregate (ID 8) from the ARTS SSDB is used.

The question that we aim to address here is whether the combination of active and passive observations is able to constrain both the size and concentration of the ice particles in the cloud. To investigate this, the $N_{0}^{*}$ and $D_{\mathrm{m}}$ parameters of the homogeneous cloud layer are varied, and observations of the cloud are simulated. The cloud signal in the radiometer observations is the difference between the cloudy- and clear-sky brightness temperatures $\left(\Delta T_{\mathrm{B}}\right)$. The signal in the active observations is here defined as the maximum of the measured profile of radar reflectivity $\mathrm{dBZ}_{\max }$. Figure 5 displays the contours of $\Delta T_{\mathrm{B}}$ and $\mathrm{dBZ}_{\max }$ with respect to $D_{\mathrm{m}}$ and the cloud's water content, which is proportional to $N_{0}^{*}$ as follows:

$\mathrm{WC}=\frac{\pi \rho}{4^{4}} N_{0}^{*} D_{\mathrm{m}}^{4}$,

with $\rho$ the density of ice.

Along the $\mathrm{dBZ}_{\max }$ contours the cloud composition changes, but the observed signal stays the same. This shows the ambiguity of the radar observations with respect to the cloud composition. A necessary condition for a passive observation at a given frequency to be able to resolve this ambiguity is that the contours of the active and passive signals cross each other. The panels in Fig. 5 thus provide an indication as to what extent the information in the radar measurement and the corresponding passive radiometer channel provide complementary information on the 2 two degrees of freedom of the PSD. The results show that the MWI channels provide complementary information only for very dense clouds consisting of large particles. In contrast to that, the ICI observations exhibit crossing contours already at lower water content and $D_{\mathrm{m}}$ values, indicating complementary information for less dense clouds and smaller particles.

\subsection{Retrieval results}

To assess the performance of the combined cloud retrieval, the developed algorithm has been applied to the two designated cloud scenes. The same retrievals have been performed with a radar-only and a passive-only version of the algorithm to serve as baselines for the evaluation of the combined retrieval. Each retrieval was performed multiple times using the different ice particle models listed in Table 4 . Since the results for both test scenes are qualitatively similar, results from the second scene are provided in Appendix A. Complete results for all retrieval quantities, test scenes and particle shapes are provided in the Supplement to this article.

The simulated observations which were generated to test the retrievals are shown for the first test scene in Fig. 6. Independent Gaussian noise with standard deviations according to sensor specifications has been added to the simulated observations to account for sensor noise. It is important to note that the simulated observations used to test the retrieval assume different microphysics than what is assumed in the retrieval. The synthetic observations are computed using the six hydrometeor classes from the GEM model, while the retrieval forward model assumes only two classes of hydrometeors.

\subsubsection{Water content}

Retrieved IWC obtained using the Large Plate Aggregate particle model for the first test scene is displayed together with the reference IWC field in Fig. 7. The reference IWC is defined here as the sum of the masses of the four frozen hydrometeor species in the GEM model scenes.

The normalized $\chi_{y}^{2}$ values of the three retrieval configurations, displayed in panel (a), give an indication of how well the retrievals are able to fit the observations. For the radaronly retrieval, the values are much smaller than 1 for most parts of the scene, while for the passive-only and combined retrieval they are around the expected value of 1 . This indicates that the radar-only retrieval overfits the observations, while the passive-only and combined retrievals achieve the expected fit. The exception is the region around $3^{\circ} \mathrm{N}$, where the cloud is particularly thick and consists of a mix of different hydrometeor types. Here especially, the passive-only retrieval has problems fitting the observations.

In terms of ice water path (IWP), all methods provide fairly good estimates of the reference values with the combined retrieval consistently yielding the smallest deviations. Larger differences between the methods are observed when comparing the retrieval results in terms of IWC. While the vertical structure of the cloud is captured only very roughly by the passive retrieval, it is better resolved by the radar-only and the combined retrieval. On closer inspection, however, it becomes evident that the radar-only retrieval deviates systematically from the reference IWC in specific regions of the cloud, such as for example the upper part of the cloud between 0 and $2^{\circ} \mathrm{N}$. These deviations are corrected in the results from the combined retrieval; however certain retrieval artifacts remain visible.

For a more quantitative assessment of the retrieval performance, retrieved water content is plotted against the reference water content in Fig. 8. In terms of precision, the passive-only retrieval performs worst, while both the radaronly and combined retrieval yield much smaller spread in the retrieved values. This is not surprising considering that the passive observations do not contain sufficient information on the vertical distribution of IWC to yield accurate results at the resolution of the model scenes. In terms of overall accuracy, i.e., systematic deviations from the diagonal, no clear differences between the three configurations are visible. However, the color-coding with respect to hydrometeor species reveals that the radar-only retrieval is biased for specific hydrometeor classes. In the combined and even the passive-only results, this effect is weaker, and the clusters are generally moved towards the diagonal. For graupel, all retrievals perform badly, but this is likely due to it being present only in 

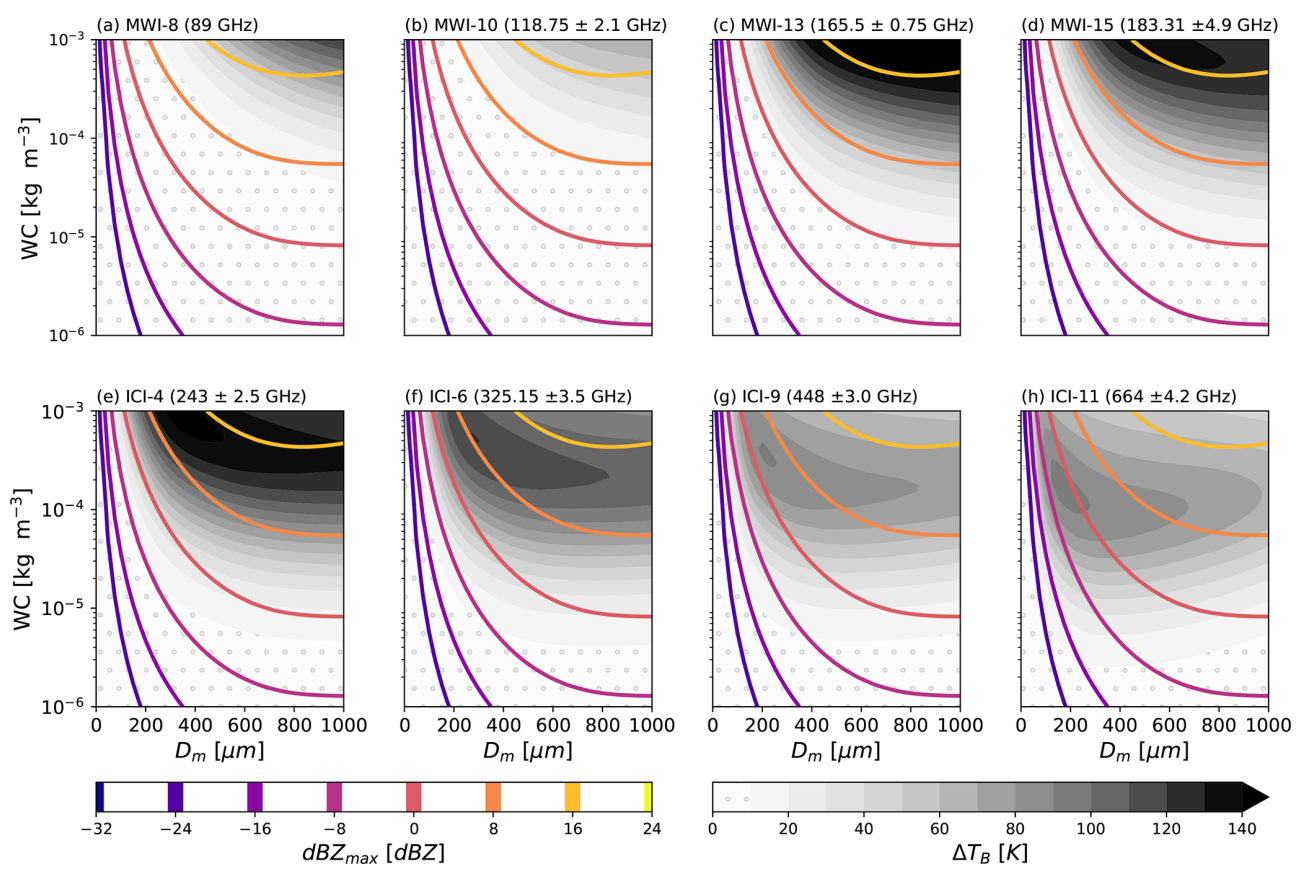

Figure 5. Simulated observations of a homogeneous, $5 \mathrm{~km}$ thick ice cloud centered at $10 \mathrm{~km}$ with varying water content $m$ and mass-weighted mean diameter $D_{\mathrm{m}}$. The panels display the maximum radar reflectivity $\mathrm{dBZ}_{\mathrm{max}}$ overlaid onto the cloud signal $\Delta T_{\mathrm{B}}$ measured by selected radiometer channels of the MWI (first row) and ICI radiometers (second row).

(a) Total hydrometeor content

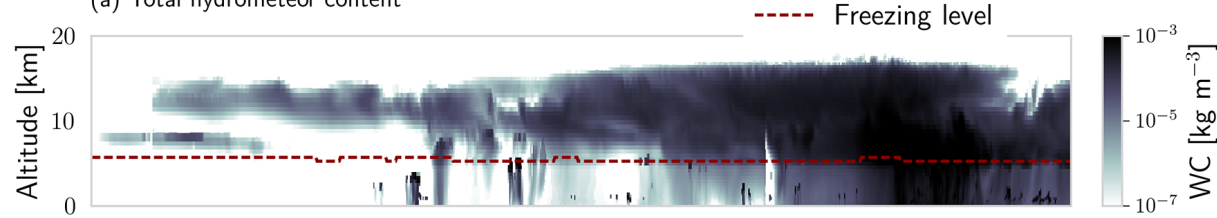

(b) Radar observations

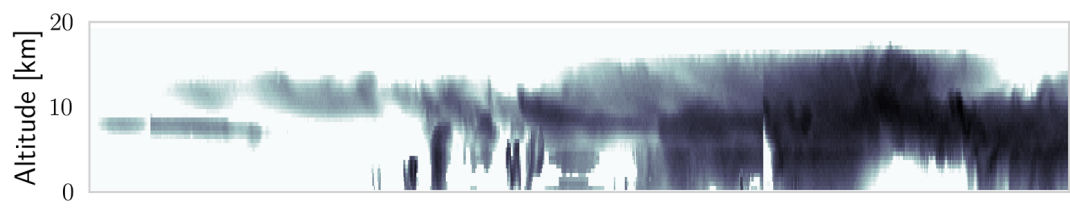

(c) Radiometer observations

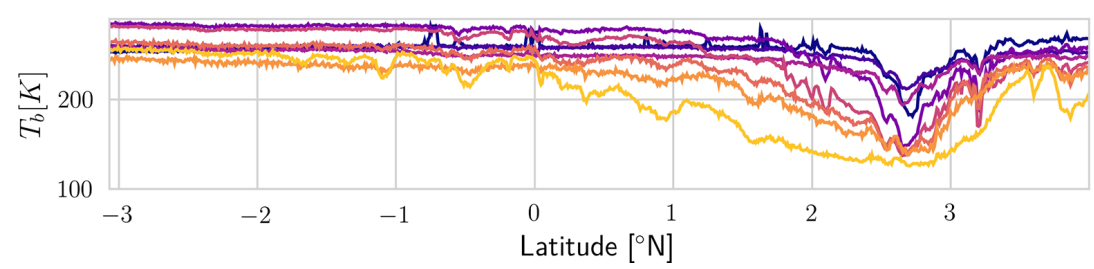

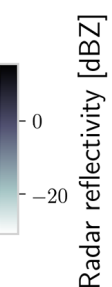

MWI-8 $(89 \mathrm{GHz})$

- MWI-10 $(118.75 \pm 2.1 \mathrm{GHz})$

- MWI-13 $(165.5 \pm 0.75 \mathrm{GHz})$

- MWI-15 $(183.31 \pm 4.9 \mathrm{GHz})$

- ICl-4 $(243 \pm 2.5 \mathrm{GHz})$

— ICI-6 $(325.15 \pm 3.5 \mathrm{GHz})$

$-\mathrm{ICl}-9(448 \pm 3.0 \mathrm{GHz})$

$-\mathrm{ICl}-11(664 \pm 4.2 \mathrm{GHz})$

Figure 6. Total water content (WC) and simulated observations for the first test scene. Panel (a) displays the total water content, i.e., the sum of the water content of all hydrometeor species of the GEM model. Panel (b) shows the simulated radar reflectivities. Panel (c) displays the simulated brightness temperatures for a selection of channels of the MWI and ICI radiometers. 


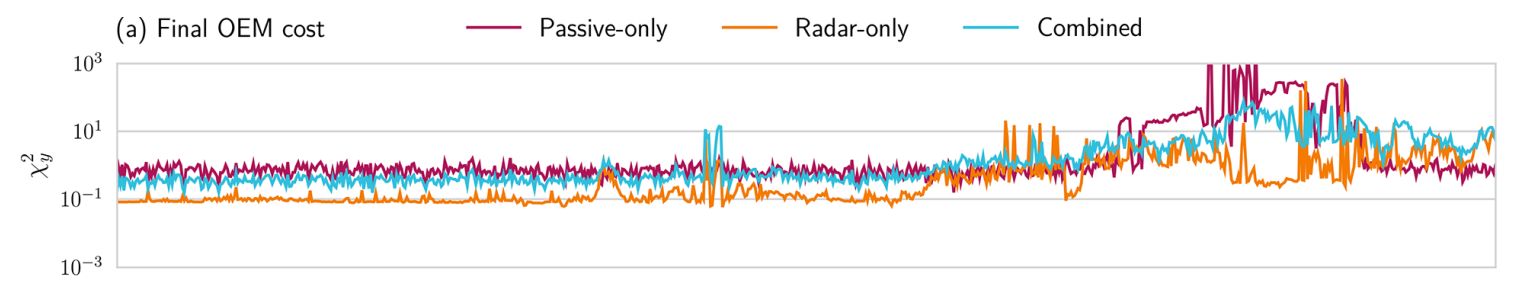

(b) IWP

Snow Ice Graupel Hail

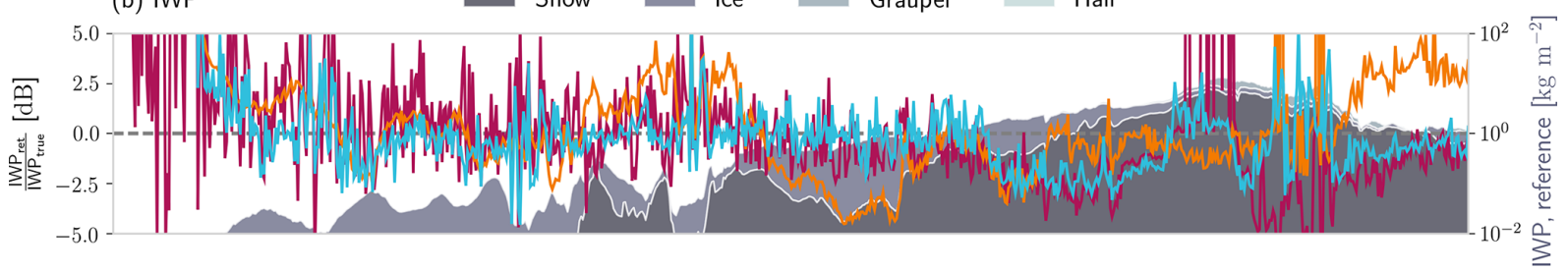

(c) Reference

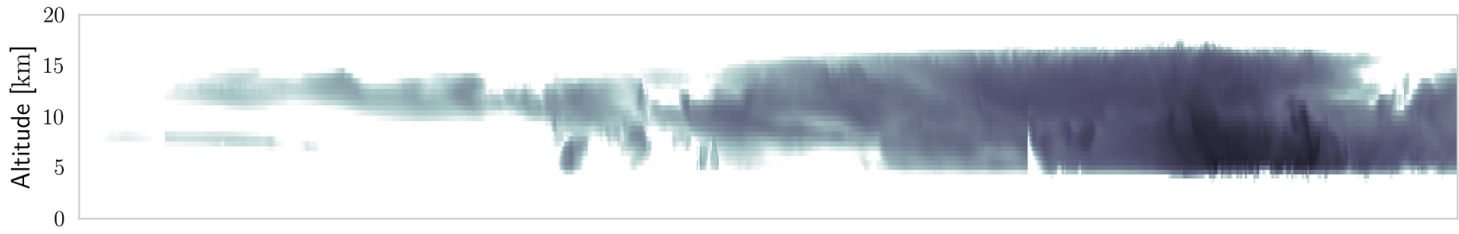

(d) Passive-only

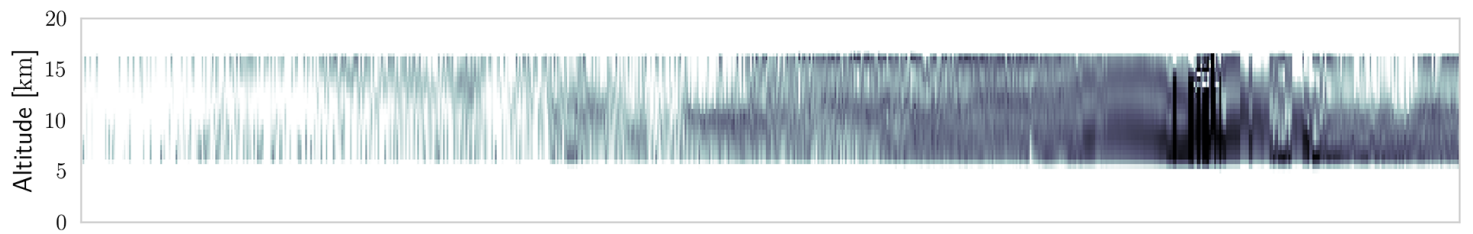

(e) Radar-only

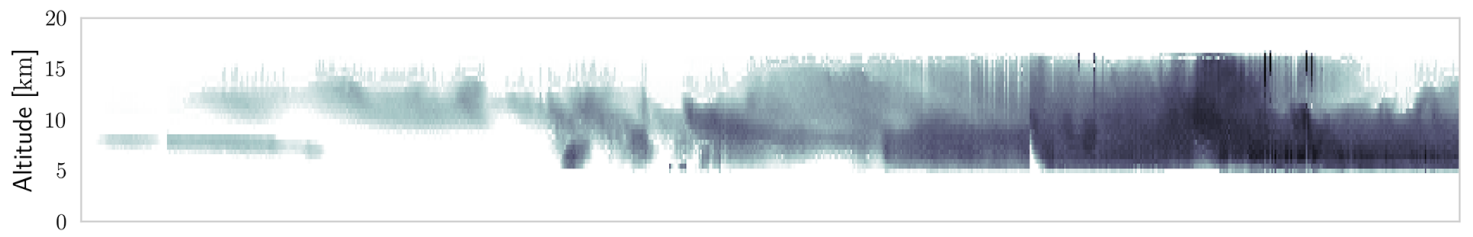

(f) Combined

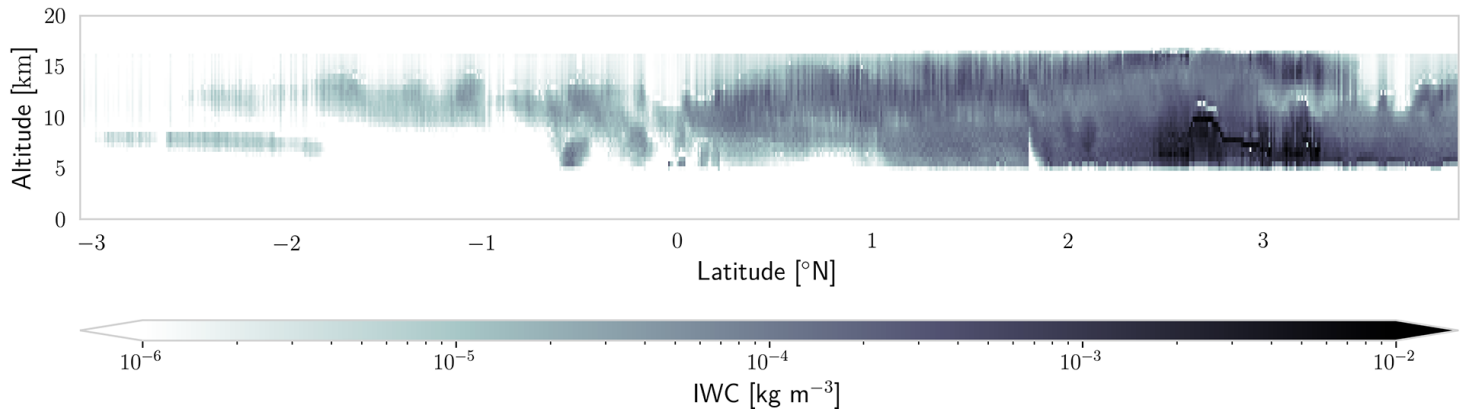

Figure 7. Results of the ice hydrometeor retrieval for the first test scene using the Large Plage Aggregate particle model. Panel (a) displays the value of the $\chi_{y}^{2}$ diagnostic normalized by the dimension of the measurement space of the corresponding retrieval. Panel (b) displays retrieved IWP in decibels $(\mathrm{dB})$ relative to the reference IWP. Reference IWP and the contributions from different hydrometeor classes are displayed by the filled areas in the background. Panel (c) shows the reference IWC from the model scene. Panels (d), (e) and (f) display the retrieval results for the passive-only, radar-only and combined retrieval, respectively. 

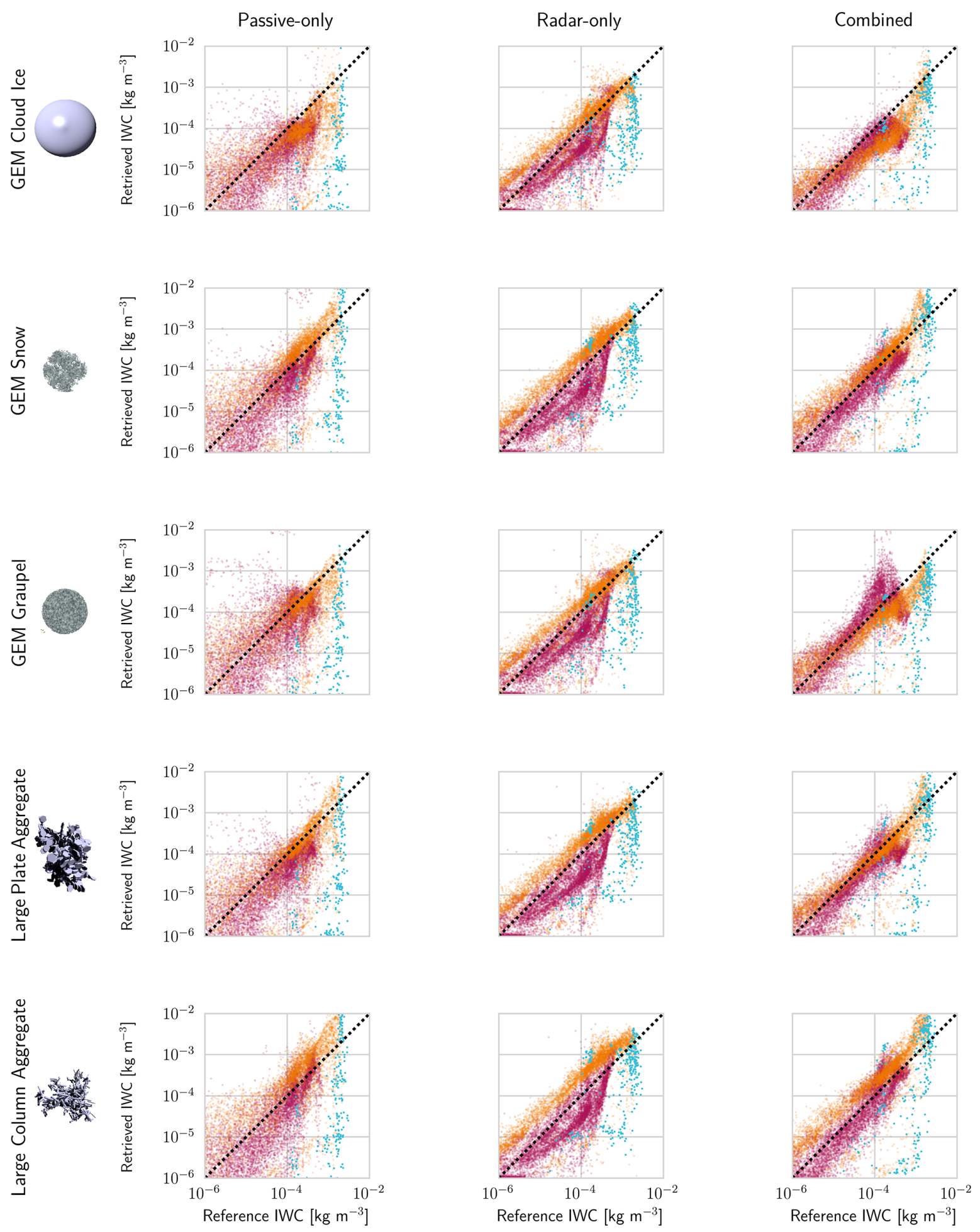

Figure 8. Retrieved IWC plotted against reference IWC for the tested retrieval configurations. Each row shows the retrieval results for the particle shape shown in the first panel. The following panels show the retrieval results for the passive-only (first column), the radaronly (second column) and the combined retrieval (third column). Markers are colored according to the prevailing hydrometeor type at the corresponding grid point in the test scene. Due to their sparsity, markers corresponding to graupel are drawn at twice the size of the other markers. 
the core of the convective system where the signals from all sensors can be expected to be saturated.

Comparing the results for different particle models, a clear dependency is visible in the passive-only and the combined results, while the radar-only retrieval is affected the least. For the combined and passive-only retrieval, the effect is consistent across the methods, with the GEM Cloud Ice and Large Column Aggregate yielding the largest deviations and the Large Plate Aggregate yielding the most accurate results.

To summarize retrieval performance for all tested retrieval methods and particle shapes, the distributions of the logarithmic error

$$
E_{\log _{10}}=\log _{10}\left(\frac{x_{\text {retrieved }}}{x_{\text {reference }}}\right)
$$

for the retrieved IWC and IWP are displayed in Fig. 9. In addition to the two-moment version of the radar-only retrieval, this figure also displays results of the single-moment version of the retrieval, which was found to yield better IWC retrievals for the second test scene.

The error for IWC has been computed considering only grid points where either reference or retrieved IWC is larger than $10^{-6} \mathrm{~kg} \mathrm{~m}^{-3}$. Similar to the results presented above, the combined retrieval yields the smallest retrieval errors for suitable choices of the particle model. Although the twomoment, radar-only retrieval performs similar to the combined retrieval in terms of precision, it yields significant systematic errors for the second scene. The reason for this can be understood considering the cloud composition displayed in Fig. 1. Since the clouds in the second test scene consist mostly of snow, the bias of the radar-only retrieval with respect to this specific hydrometeor species (cf. Figs. 8 and A2) leads to the large observed systematic errors for the second scene. The single-moment, radar-only retrieval does not produce the same large systematic errors for the second scene but instead produces systematic errors for the first scene. The passive-only retrieval yields the largest errors in terms of retrieved IWC due its low vertical resolution.

In terms of IWP, however, the errors of the passive-only retrieval are decreased making the retrieval comparable to the other methods. For the radar-only and combined retrievals, the precision is generally increased, but the systematic deviations observed for IWC persist. This leads, particularly for the second test scene, to significant systematic errors in the IWP retrieved by the two-moment, radar-only retrieval.

Also in these results, a strong dependence on the applied particle model is observed for the passive-only and combined retrievals. The errors are particularly large for the GEM Cloud Ice and the Large Column Aggregate. Although the impact is stronger for the M1 version, the particle shape has less impact on the retrieval performance of the radar-only retrieval and does not affect the large systematic errors observed for the second test scene.

\subsubsection{Particle number concentrations}

Particle number concentrations of frozen hydrometeors have been derived from the retrieved $N_{0}^{*}$ and $D_{\mathrm{m}}$ parameters by computing the zeroth moment of the corresponding PSD. The resulting particle number concentration fields are displayed together with the reference field in Fig. 10. To simplify the comparison, number concentrations are displayed only where the corresponding reference or retrieved IWC is larger than $5 \times 10^{-6} \mathrm{~kg} \mathrm{~m}^{-3}$.

Comparing the passive-only and the radar-only retrieval to the reference fields shows that both methods have little to no skill in predicting number concentrations. Although the passive-only retrieval partly captures the gradient between very high concentrations at the top of the cloud and the low concentrations at the bottom, it is not at all resolved in the radar-only retrieval.

In contrast to this, the combined retrieval manages to reproduce this gradient in most parts of the scene. The strongest deviations of the combined results from the reference field are observed between 2 and $3^{\circ} \mathrm{N}$ latitude. Here, the results strongly underestimate the true number concentrations. Comparison with the cloud composition displayed in Fig. 1a shows that this region contains large amounts of both cloud ice and snow. The retrieval uses only a single hydrometeor species to represent ice in the atmosphere and is therefore not able to represent such heterogeneous conditions. Since snow will have a stronger impact on the observations, the retrieval in these regions will likely tend to represent snow rather than ice, which leads to the low retrieved number concentrations.

Figure 11 displays scatter plots of the reference and retrieved particle number concentrations for all three methods and two particle models from the first test scene. Markers in the plot are color coded according to their homogeneity in the reference scene, here defined as the ratio of the maximum water content of any of the frozen hydrometeor species and the total water content. These results confirm that the passive-only retrieval possesses some sensitivity to the particle number concentrations since the cluster at low concentrations corresponding to snow is placed correctly on the diagonal, which is not the case for the radar-only retrieval. The radar-only retrieval does not exhibit any retrieval skill, hardly reproducing any of the variation in the reference values. Contrary to this, the combined retrieval moves both clusters, the one corresponding to snow and the one at high number concentrations corresponding to cloud ice, towards the diagonal. This indicates that it is capable of distinguishing the microphysical properties of cloud ice and snow. Furthermore, the color coding shows that the strongest deviations between retrieved and reference number concentrations occur for grid points where the cloud composition is heterogeneous.

The general effect of particle shape on the retrieval results is similar to what has been observed for IWC, which is why only results for two particle shapes are shown. For the 
(a) IWC, first scene

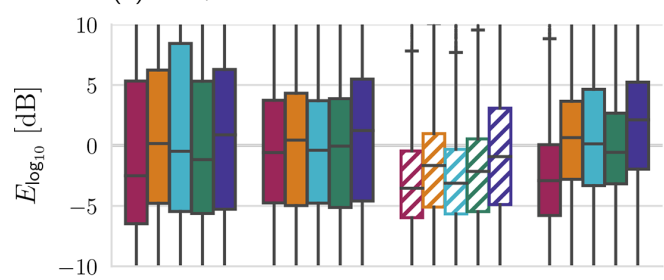

(c) IWP, first scene

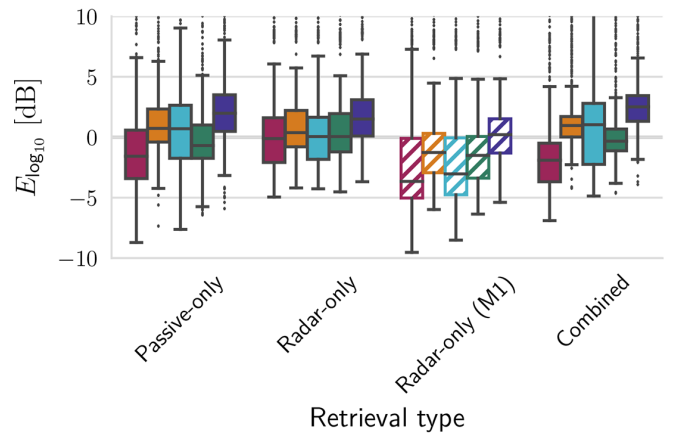

$\begin{array}{ll}\text { GEM Cloud Ice } & \text { GEM Graupel } \\ \text { GEM Snow } & \text { Large Plate Aggregate }\end{array}$ (b) IWC, second scene

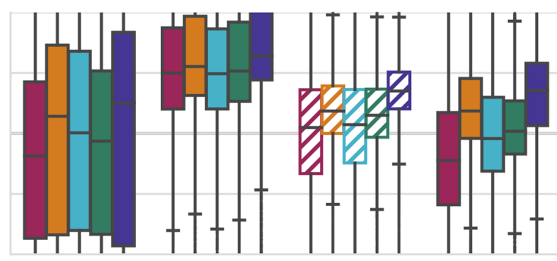

(d) IWP, second scene

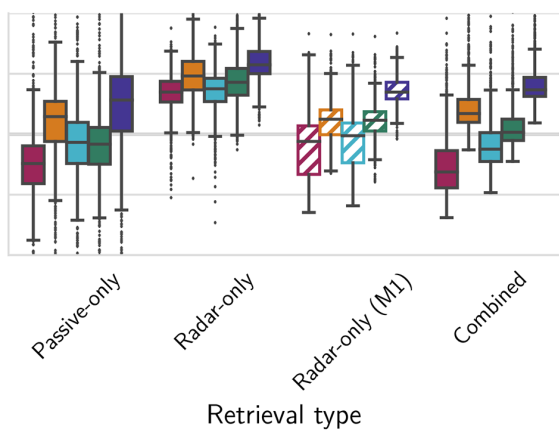

Large Column Aggregate

Figure 9. Distributions of the logarithmic retrieval error in IWC and IWP for all tested retrieval methods and particle shapes displayed as box plots. Colored boxes display the interquartile range (IQR), while whiskers show the full range of all points not considered outliers. Points whose distance to the IQR is larger than 1.5 times the width of the IQR are considered outliers and drawn as markers. Two results are shown for the radar-only retrieval, one for the standard version retrieving both PSD moments (solid boxes) and one for the single-moment (M1) version (diagonal hatches).

passive-only and combined retrieval, the GEM Cloud Ice and Large Column Aggregate models yield the worst retrieval results, while the Large Plate Aggregate performs best. For the radar-only retrieval no noticeable differences are observed between different particle models.

\subsubsection{Information content}

To quantify the information content of the single-instrument and combined observations, the degrees of freedom for signal (DFS) have been computed following Rodgers (2000) by calculating the trace of the averaging kernel matrix

$\mathbf{A}=\left(\mathbf{K}^{T} \mathbf{S}_{\mathrm{e}}^{-1} \mathbf{K}+\mathbf{S}_{\mathrm{a}}^{-1}\right)^{-1} \mathbf{K}^{T} \mathbf{S}_{\mathrm{e}}^{-1} \mathbf{K}$,

where $\mathbf{K}=\frac{\mathrm{d} \mathbf{F}(\boldsymbol{x})}{\mathrm{d} \boldsymbol{x}}$ is the Jacobian of the forward model. The information content and its decomposition into contributions from different retrieval quantities are displayed in Fig. 12.

With respect to ice, the passive-only retrieval yields the lowest information content. For the radar-only retrieval, the information content is significantly higher, on the order of 20 degrees of freedom, but the major part of it is attributed to the $D_{\mathrm{m}}$ parameter. For the combined retrieval, the total information content on ice hydrometeors is increased compared to the radar-only retrieval in regions where the passive-only retrieval provides information on frozen hydrometeors. In ad- dition to that, a clear shift in information content from $D_{\mathrm{m}}$ to $N_{0}^{*}$ can be observed over both scenes.

The information content for rain is much smaller, but in relative terms the general behavior is the same as for ice. For $\mathrm{RH}$, no difference is observed for the information content provided by the passive-only and combined retrievals. For CLWC the information content of the combined observations is increased slightly but remains limited to a few degrees of freedom.

In order to allow a more detailed analysis of the complementarity of the information in the passive and active observations, Fig. 13 displays the ratio of the DFS of the combined retrieval and the sum of the DFS in the radarand passive-only retrievals. Comparison with the information content provided by the radar-only observations confirms that the active and passive observations consistently provide a fairly high amount of complementary information across both scenes.

\subsubsection{Impact of assumed ice particle shape}

The impact of the assumed ice particle shape on the retrieval results raises the question whether it also affects the quality of the fit to the observations. To investigate this, the residuals for the radar observations and three ICI channels are displayed in Fig. 14. Each test scene contains a region where the 
(a) Reference

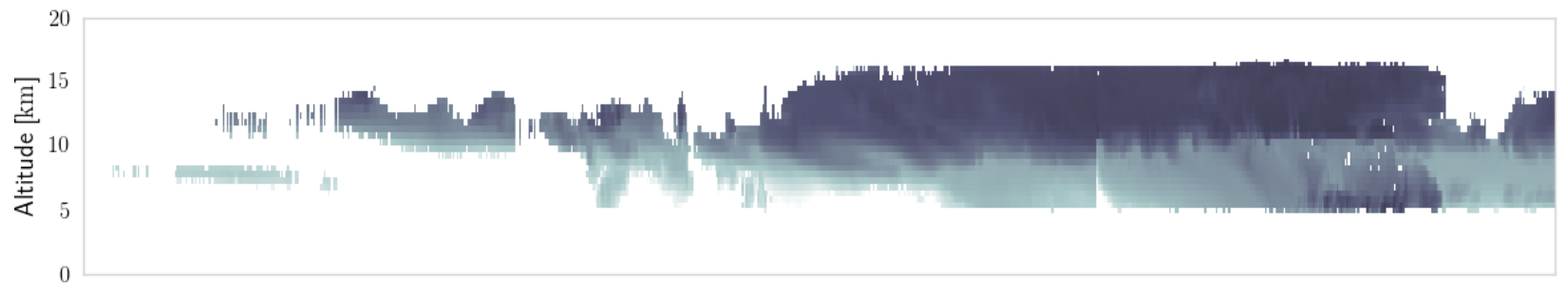

(b) Passive-only

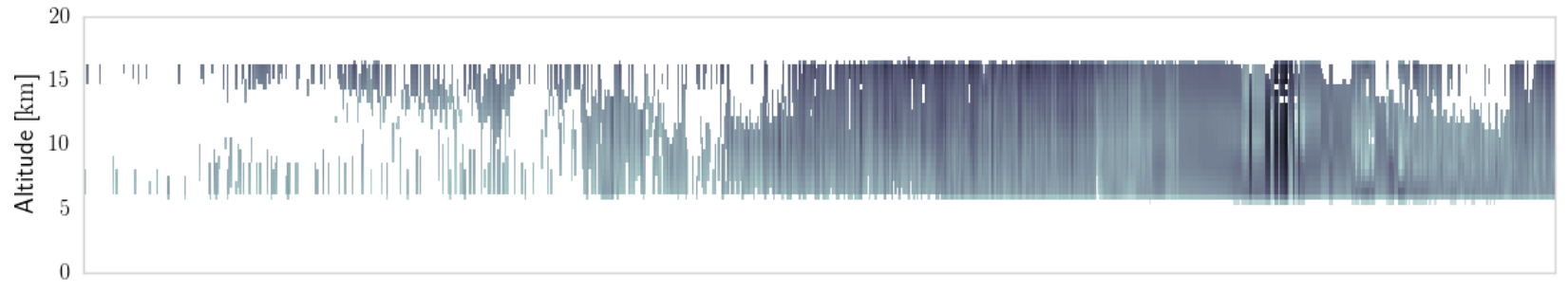

(c) Radar-only

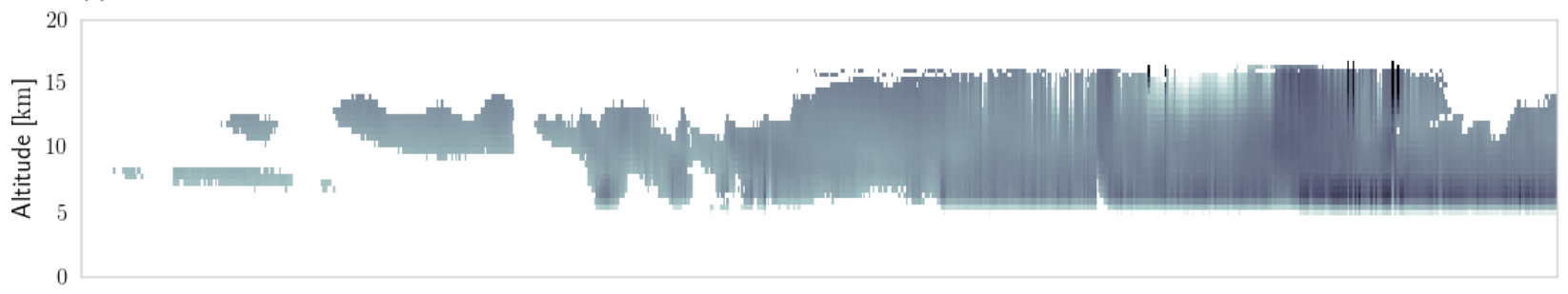

(d) Combined

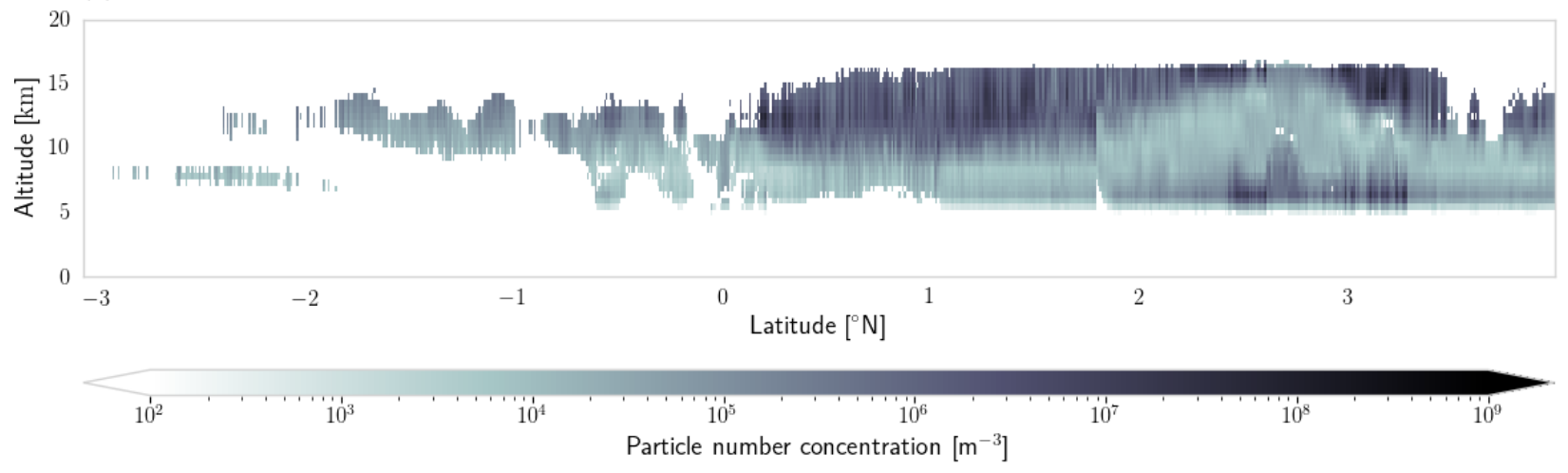

Figure 10. Reference and retrieved particle number concentrations of frozen hydrometeors for the first test scene obtained with the Large Plate Aggregate particle model. Panel (a) displays the reference number concentrations from the model scene. Panels (b), (c) and (d) display the retrieval results for the passive-only, radar-only and combined retrieval. Only values for which the corresponding reference or retrieved IWC was larger than $5 \times 10^{-6} \mathrm{~kg} \mathrm{~m}^{-3}$ are shown here.

retrieval does not fit the observations well and where substantial deviations between the fitted and true observations are observed. It is also in these regions that the fits obtained with different particle models differ. These are both regions where the cloud is very thick and both the radar and passive observations are likely saturated. Since these are difficult regions for the retrieval, it is not clear whether these differences can be related directly to the assumed particle shape. In contrast to this, the retrieval fits the observations well in the remain- ing parts of the scene. The exception is the GEM Graupel particle, for which significant misfits are observed in the first test scene between 0 and $1^{\circ} \mathrm{N}$ latitude.

\subsubsection{Humidity and cloud water}

The developed passive and combined retrieval algorithms also retrieve profiles of RH and CLWC. For RH, both retrievals demonstrate sensitivity, but no improvement was ob- 

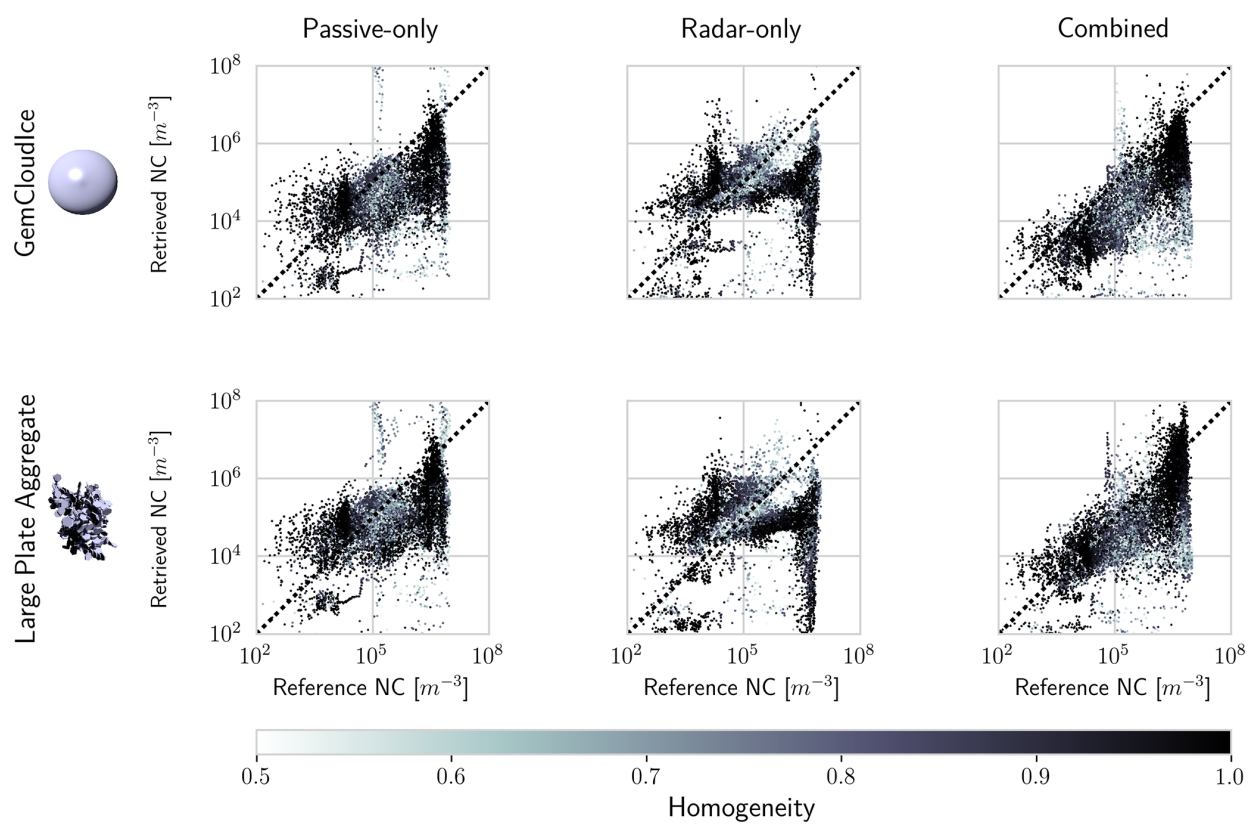

Figure 11. Scatter plots of the retrieved particle number concentration (NC) at grid points with reference IWC larger than $10^{-5} \mathrm{~kg} \mathrm{~m}^{-3}$ for two different particle models. Rows show the results for the different particle models used in the retrieval, while columns display results for different retrieval methods. The marker color encodes the homogeneity of the corresponding ice mass, which is computed as the ratio of the maximum water content of any of the frozen hydrometeor species and total IWC.

(a) Scene 1, passive-only

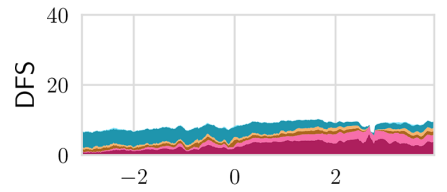

(d) Scene 2, passive-only

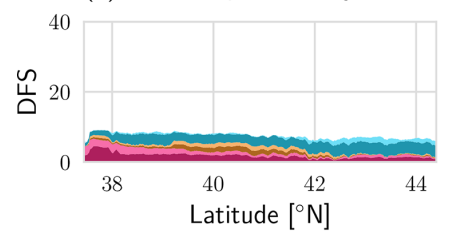

(b) Scene 1, radar-only

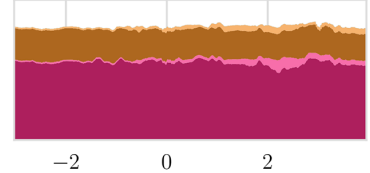

(e) Scene 2, radar-only

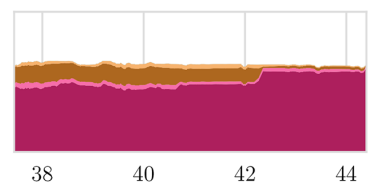

(c) Scene 1, combined

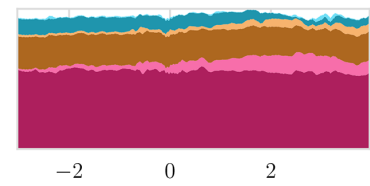

(f) Scene 2, combined

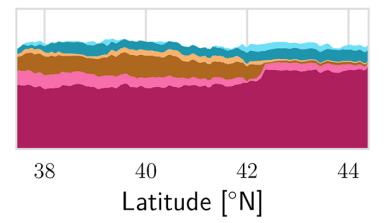

$\begin{array}{lll}\text { Ice } D_{m} & \text { Rain } D_{m} & \mathrm{RH} \\ \text { Ice } N_{0}^{*} & \text { Rain } N_{0}^{*} & \mathrm{CLWC}\end{array}$

Figure 12. Information content in terms of DFS using Large Plate Aggregate for all retrieval configurations and both test scenes. The colored areas in each plot represent the contribution to the cumulative degrees of freedom from each retrieval quantity. Results for the first and second test scene are displayed in the first (a-c) and second row (d-f), respectively. The first (a, d), second (b, e) and third panel (c, f) in each row show the results for the passive-only, radar-only and the combined retrieval.

served in the results of the combined retrieval compared to the passive-only retrieval.

Results of the CLWC retrieval are shown in Fig. 15. For the retrieved CLWC, the combined retrieval yields slightly improved results compared to the passive-only retrieval. The improvements are observed mostly in the retrieved cloud liquid water path (CLWP) in the northern part of the scene. It should be noted that the cloud in this part is a mixed-phase cloud and that both retrievals successfully retrieve IWC and CLWC. At the center of the scene both retrievals fail to retrieve the CLWC. The reason for this seems to be that rain is present in these regions whose signal cannot be separated by the retrieval from that of the liquid cloud droplets. 
(a) First scene

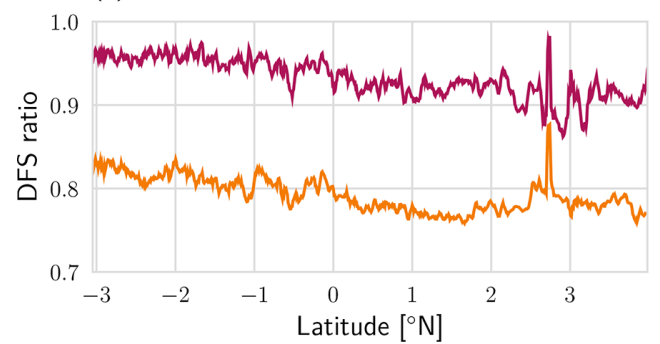

(b) Second scene

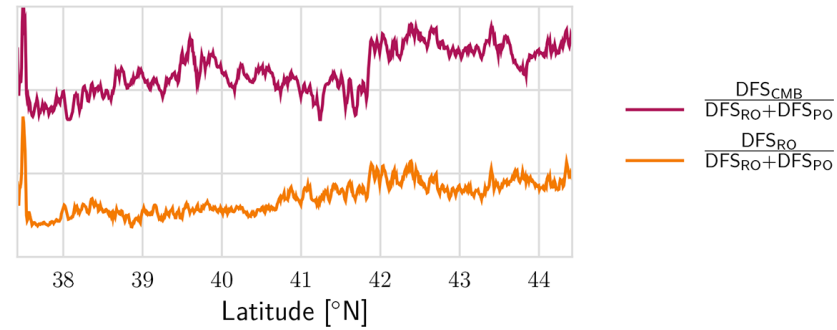

Figure 13. DFS ratios of the combined retrieval $\left(\mathrm{DFS}_{\mathrm{CMB}}\right)$ and the sum of the DFS of the single-instrument retrievals $\left(\mathrm{DFS}_{\mathrm{RO}}+\mathrm{DFS}_{\mathrm{PO}}\right)$ as well as the radar-only retrieval and the sum of the DFS of the single-instrument retrievals for the two test scenes.

(a) First scene, radar reflectivities

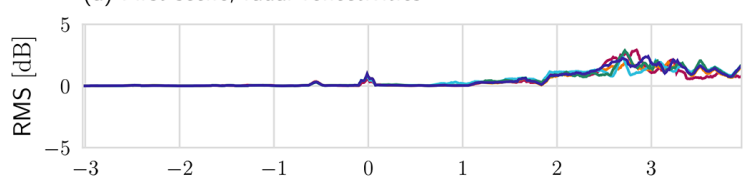

(c) First scene, $183.31 \pm 7 \mathrm{GHz}$

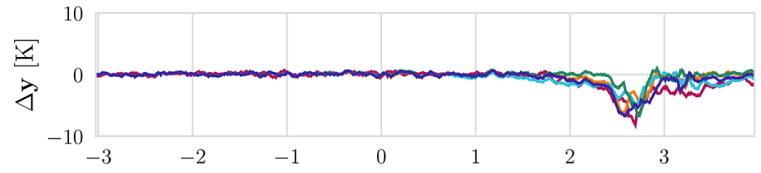

(e) First scene, $325.15 \pm 3.5 \mathrm{GHz}$

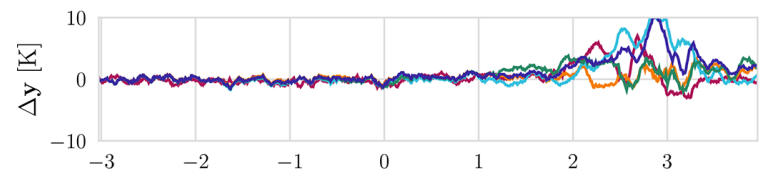

(g) First scene, $664 \pm 4.2 \mathrm{GHz}$

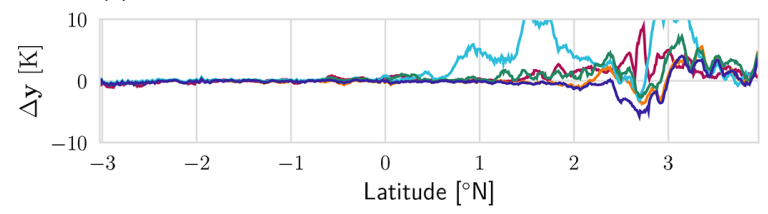

- GEM Cloud Ice

- GEM Snow (b) Second scene, radar reflectivities

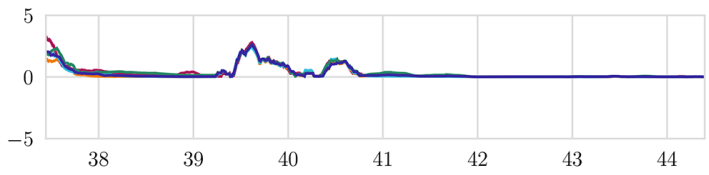

(d) Second scene, $183.31 \pm 7 \mathrm{GHz}$

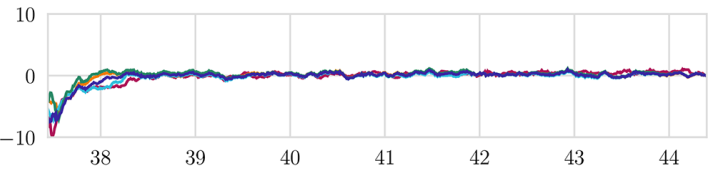

(f) Second scene, $325.15 \pm 3.5 \mathrm{GHz}$

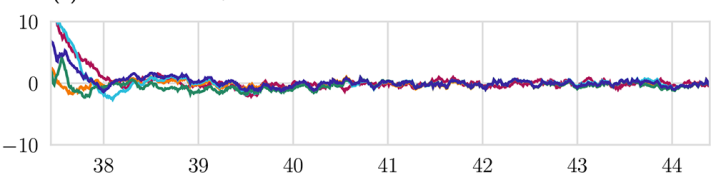

(h) Second scene, $664 \pm 4.2 \mathrm{GHz}$

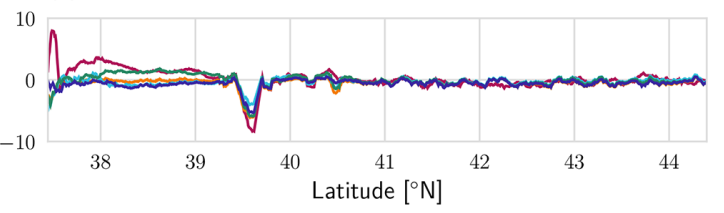

- Large Column Aggregate

Figure 14. Residuals of the fitted observations. First row (a, b) shows the profile root-mean-squared error (RMS) between fitted $(\hat{\boldsymbol{y}})$ and true (y) radar observations for the two test scenes. Rows $2(\mathbf{c}, \mathbf{d}), 3(\mathbf{e}, \mathbf{f})$ and $4(\mathbf{g}, \mathbf{h})$ show the residual $\Delta \boldsymbol{y}=\hat{\boldsymbol{y}}-\boldsymbol{y}$ for a selection of ICI channels.

\section{Discussion}

The principal aim of this study was to investigate the synergies between radar and passive sub-millimeter observations for the retrieval of frozen hydrometeors. To this end, a simplified numerical experiment has been presented that demonstrates the existence of complementary information on the microphysical properties of ice clouds in the radar and passive microwave observations. Furthermore, a combined retrieval algorithm has been developed to demonstrate the feasibility of the synergistic retrieval and further explore its potential as well as current limitations.
The novelty of this work lies, in part, in the application of ICI's sub-millimeter channels, which sets it apart from the combined retrievals developed for the TRMM and GPM missions. Moreover, the development of a fully consistent variational retrieval in which all retrieval quantities are retrieved simultaneously using the observations from all sensors is a key aspect of this study. This allows comparison of the combined retrieval to equivalent radar-only and passive-only configurations and therefore a direct analysis of the synergies between the active and passive observations. 
(a) Liquid water path

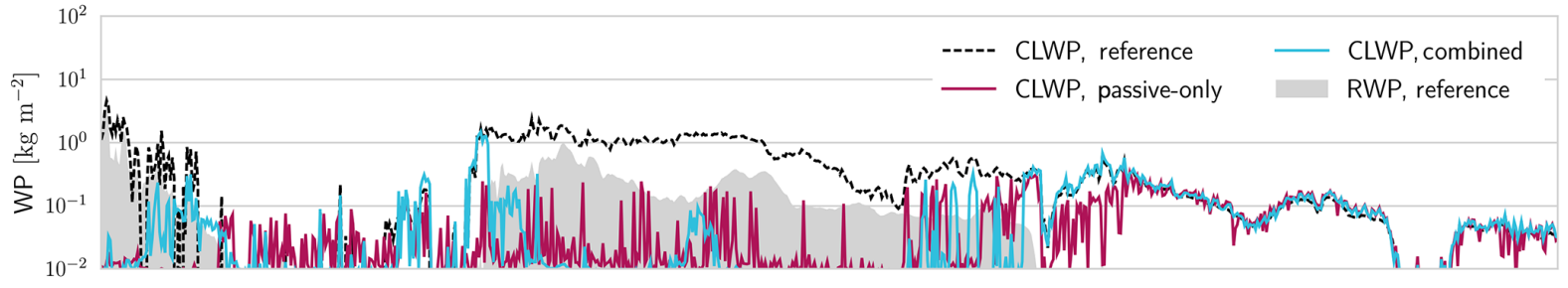

(b) Reference

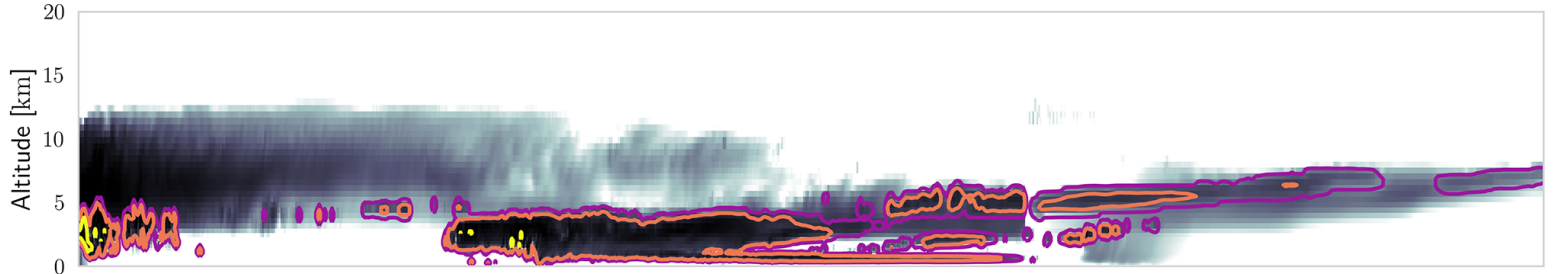

(c) Passive-only

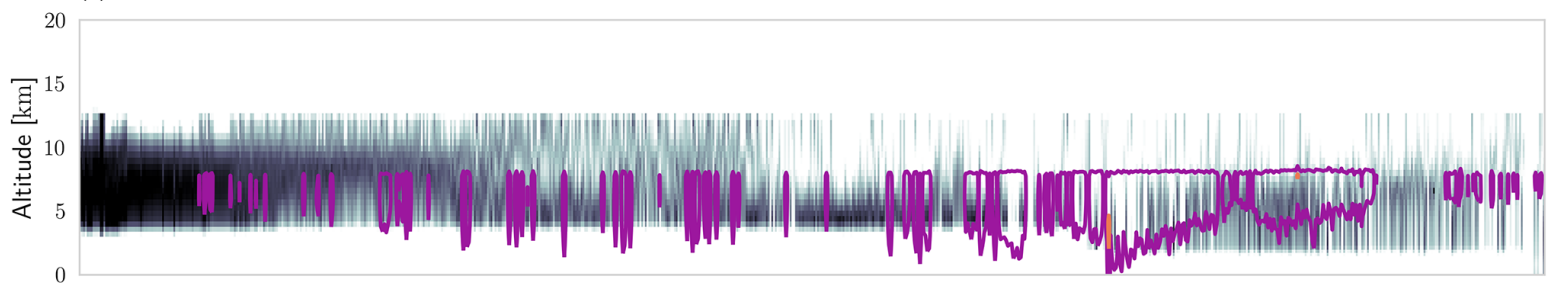

(d) Combined

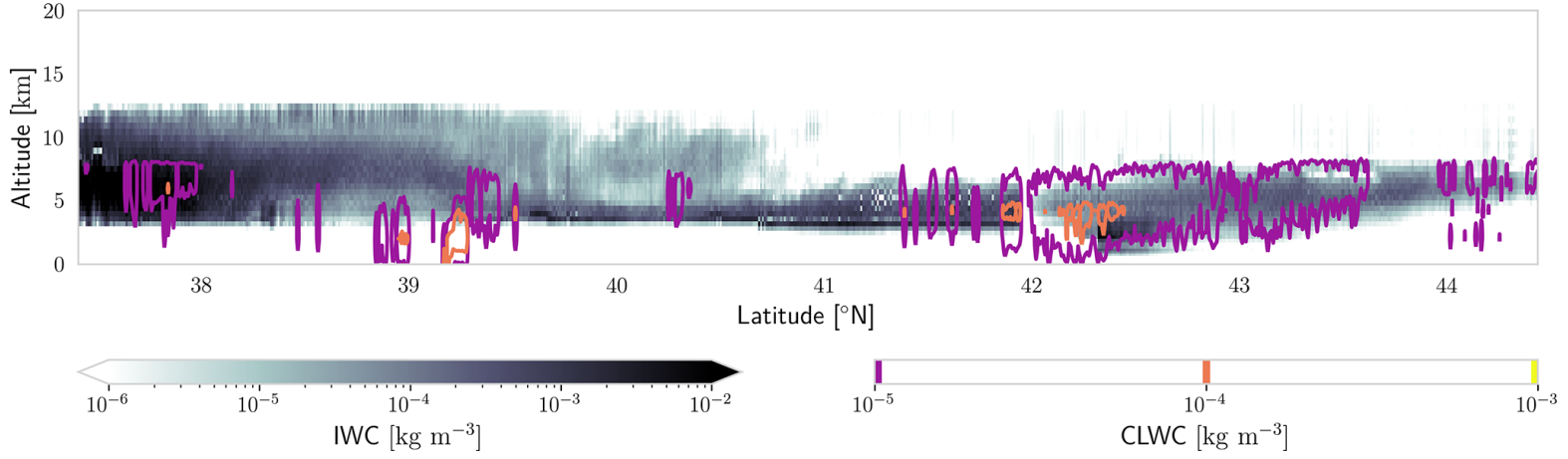

Figure 15. Reference and retrieved cloud liquid water path (CLWP), rain water path (RWP), CLWC and IWC. Panel (a) shows the reference and retrieved CLWP for each profile. Panel (b) displays reference CLWP contours drawn on top of the total hydrometeor content. Retrieval results for passive-only and combined retrieval are given in panels (c) and (d).

\subsection{Fundamental synergies}

The experiment presented in the first part of this study aimed to illustrate the fundamental synergies of active and passive microwave observations. It compared the cloud signals observed by a radar, a millimeter-wave radiometer and a sub-millimeter-wave radiometer. The results indicate that the combined observations can constrain the size and concentration of particles in the cloud. However, the complementary information content between the active and passive observations depends on both the properties of the observed cloud and the frequency of the observations. For the lower frequen- cies considered in this study, i.e., the highest-frequency channels of the MWI radiometer, the regions where both observations provide complementary information on the particle size distribution of the cloud are limited to very high water content and particle sizes. It should be noted, however, that since the radar simulations neglect multiple scattering, these results may not fully carry over to spaceborne observations.

As the passive observing frequency increases, the regions of complementary information content extend down to smaller particle sizes and lower water content. Especially the highest-frequency channels of the ICI radiometer can there- 
fore be expected to provide complementary information to a $\mathrm{W}$-band radar in a combined observation scenario.

\subsection{Combined cloud retrieval}

In the second part of the study, we have presented results from a combined, variational cloud retrieval applied to synthetic observations from two test scenes from a CRM. The results of the combined retrieval were compared to that of a passive- and a radar-only version of the retrieval algorithm. The simulated observations assumed an airborne viewing geometry and therefore neglected potential errors caused by different or non-overlapping antenna beams as well as inhomogeneity of the atmosphere across the beams. A source of forward model error was included by applying a more complex microphysics scheme in the simulations than the one used in the retrieval. This permits a rough assessment of the retrieval error caused by the simplified modeling of cloud microphysics in the retrieval.

\subsubsection{Retrieval performance}

Of the three considered retrieval implementations, the passive-only retrieval clearly performs worst in terms of retrieved IWC. It should be noted, however, that the passiveonly retrieval presented here has not been fully optimized and should therefore not be taken as representative of the potential performance of the MWI and ICI radiometers for IWC retrievals. To ensure a fair comparison, the retrieval uses almost the same a priori assumptions as the other two retrievals, which in the presented case provide only very limited information on the vertical structure of the cloud. As has also been shown by other studies, the passive observations do provide information on the vertical distribution of ice in the atmospheric column (Wang et al., 2017; Grützun et al., 2018), but the information content is limited to a few degrees of freedom. It is therefore unlikely that the vertical resolution of the passive-only retrieval can be improved drastically without further constraining it a priori, as is typically done in retrievals that use Monte Carlo integration or neural networks (Pfreundschuh et al., 2018).

With respect to IWP, however, the passive retrieval can perform as well or even better than the radar-only retrieval. Furthermore, the results in Fig. 10 indicate that the passive observations provide some information on the particle number concentrations, which is not the case for the radar observations. This shows that passive observations at multiple frequencies can constrain the microphysics better than singlefrequency radar observations alone, albeit at lower vertical resolution.

As expected, the radar-only retrieval provides much better IWC retrievals than the passive-only version. However, the results of the two-moment retrieval exhibit systematic deviations from the reference values in certain regions of the cloud. The analysis shown in Figs. 8 and A2 reveals that these are caused by systematic errors in the retrieval of specific hydrometeor species from the GEM model. Interestingly, the one-moment version of the radar-only retrieval did not produce the large errors in the second scene but produces systematic errors for the first test scene. This indicates that the a priori assumptions used in the retrieval do not provide a sufficiently good description of how the $D_{\mathrm{m}}$ and $N_{0}^{*}$ parameters of the PSD covary and that the radar-only observations alone do not constrain both of them well enough. This is also plausible from an information content perspective since the radar provides only one piece of independent information at each range gate, which is insufficient to determine the 2 degrees of freedom $\left(N_{0}^{*}\right.$ and $\left.D_{\mathrm{m}}\right)$ of the PSD. This hypothesis is confirmed by the radar-retrieved number concentration fields shown in Figs. 10 and 11. While the distribution of reference values has two modes corresponding to ice and snow, the retrieved values are nearly the same throughout the whole scene indicating that the observations themselves provide almost no information on particle concentrations.

Despite certain visible artifacts in the retrieved IWC field (Fig. 7), the combined retrieval yields the best overall performance for IWC and IWP as shown in Figs. 8 and 9 given that a suitable particle model is used. The benefit of the combined observations is even more pronounced in the retrieved number concentrations (Fig. 10). Here, the passive- and radaronly retrievals show little to no skill in retrieving the number concentrations. In contrast to this, the combined retrieval was able to reproduce the general structure of the number concentration field in regions where the cloud composition is homogeneous (Fig. 11). This shows that the combined retrieval is able to distinguish the microphysical properties of ice and snow in the test scenes. Instead of relying on the a priori, the combined retrieval can use information from the observations to constrain the cloud microphysics, which avoids the systematic errors observed in the radar-only retrievals.

The a priori assumptions used in this study were chosen similar to those of the DARDAR-CLOUD retrieval since they represent well-established and validated assumptions for ice cloud retrievals. The role of the a priori is to complement the observations with additional information required to make the retrieval problem tractable. For the hydrometeor retrieval this means that the a priori determines how information from the observations, which alone is insufficient to determine both degrees of freedom of the PSD, is distributed between its $D_{\mathrm{m}}$ and $N_{0}^{*}$ parameters. For the radar-only retrieval, this works well for cloud systems containing both ice and snow but leads to biased retrievals of both IWC and IWP when this is not the case (Fig. 9). The DARDAR product resolves the ambiguity of the radar-only observations by combining the observations with colocated lidar measurements. Our results show that a similar effect can also be achieved by combining the radar with passive microwave radiometers. However, these two different types of synergies will generally be effective in different regions of the cloud: while the overlap between lidar and radar is restricted to relatively thin 
clouds and cloud tops (for a down-looking configuration), microwave radiometers will provide sensitivity further down in the cloud where particles are larger and the water content higher.

\subsubsection{Impact of the assumed particle shape}

Our experiments show a stronger sensitivity to the assumed ice particle shape for the passive-only and the combined retrievals than the radar-only retrieval. The passive observations probe the particle at multiple frequencies, and their sensitivity to particle shape, especially of the sub-millimeter channels, has been highlighted in several studies (e.g., Fox et al., 2019; Ekelund et al., 2020).

Only the combined retrieval was able to yield accurate IWC retrievals for both test scenes for suitable choices of the particle model. However, if an unsuitable particle shape is chosen, the induced errors may outweigh the benefits of the combined retrieval as is the case for the Large Column Aggregate and the GEM Cloud Ice shapes (Fig. 9). Judging from the particle properties displayed in Fig. 4, a likely explanation for the good performance of the Large Plate Aggregate and the GEM Graupel particle is that their properties are intermediate to those of GEM Cloud Ice and GEM Snow, which are the dominating shapes in the test scenes. For the test scenes considered here, this means that accurate IWC retrievals can be achieved using only a single hydrometeor species with suitable scattering properties, which are intermediate to snowflakes and heavily rimed particles.

The analysis of the residuals of the retrieval fit (Fig. 14) showed that the residuals for different particle shapes differ most where the clouds are thickest. Differences between particles are observed, but no relationship with the retrieval accuracy in terms of IWC can be established. The GEM Graupel particle, for example, yields accurate IWC retrievals but gives the worst fit for the first test scene. A likely explanation for this is that the retrieved IWC depends mostly on the overall strength of the interaction between particles and radiation for given water content, whereas the retrieval residual is likely caused by relative efficiencies at different frequencies. Moreover, in the remaining parts of the scenes, there are no differences in the residuals for different particles. This means that the retrieval can fit the observations well regardless of the assumed particle shape and indicates that the observations alone do not strongly constrain the particle shape. This makes it unlikely that particle shape can be retrieved from observations, thus requiring it to be determined a priori.

It should be noted that none of the presented retrievals account for the error caused by the simplified forward model and the choice of the particle model. This has not been pursued here because of the difficulty of fitting a suitable error model for these errors, which can be expected to be nonGaussian and scene dependent. However, it is likely that accounting for them can improve retrieval performance and weaken the impact of the particle choice on the retrieval results.

\subsubsection{Humidity and cloud water}

As an outlook, results from the CLWC retrieval have been provided despite it not being a focus of this study. Figure 15 shows improvements in retrieved CLWP and CLWC in the results of the combined retrieval compared to the passiveonly retrieval. Although the passive-only retrieval also shows sensitivity to CLWC, the results are less robust than those of the combined retrieval. This shows that combined millimeter and sub-millimeter radiometers, in particular in combination with radar observations, can be used for retrieving both frozen and cloud liquid water content in mixed-phase clouds. This conclusion is supported by the information content analysis in Figs. 12 and 13. In particular, the DFS ratio of the combined retrieval shows a distinct increase around $42^{\circ} \mathrm{N}$, where the scene contains non-precipitating, mixedphase clouds. This coincides with a slight increase in information content on CLWC in the combined compared to the passive-only retrieval shown in Fig. 12.

For the water vapor retrieval, no significant improvements in the combined retrieval results were observed, and also the analysis of the information content does not show any increase in information content. This indicates that the combined observations do not provide any direct synergies for the retrieval of humidity.

\subsubsection{Limitations}

An important limitation of this study is its scope: the aim here was not to develop a production-ready combined retrieval product but rather a proof-of-concept to explore this observational approach. The retrieval results presented here should therefore not be interpreted in absolute terms. The primary results are based on the relative performances of the three retrieval methods: given equivalent a priori assumptions, the combined retrieval demonstrates higher sensitivity to the microphysical properties than the radar-only retrieval and lower errors in terms of IWC than the passive-only retrieval.

Moreover, this study is purely based on simulations from two selected CRM scenes. These two scenes are certainly insufficient to accurately represent the variability in clouds in the atmosphere. Furthermore, the accuracy of the estimated retrieval performance will depend on the realism of the test scenes. Because of this, this study does not aim to provide an accurate assessment of the performance of the combined retrieval in absolute terms but instead a qualitative assessment of the potential of a combined retrieval based on the comparison of its results to the single-instrument retrievals.

As has been stated above, simulated observations used in this study assume a viewing geometry that is realistic only for airborne observations. They therefore do not provide a realistic assessment of the potential of a spaceborne satel- 
lite mission involving ICI, MWI and a W-band radar. For this it would be necessary to take into account a more realistic viewing geometry, beam-filling errors as well as multiple scattering in the radar observations. Quantifying the effect of these error sources on the retrieval synergies is left for future investigation.

\section{Conclusions}

The main conclusion from this work is that the combination of radar and sub-millimeter radiometer observations can, to some extent, constrain both the size and number concentration of frozen hydrometeors (Fig. 5). The increased sensitivity of the combined observations to the microphysical properties of hydrometeors helps to improve the accuracy of IWC retrievals and avoid systematic errors observed in an equivalent radar-only retrieval (Figs. 8, 9). Moreover, the combined retrieval showed clear sensitivity to particle number concentrations and was able to reproduce their vertical structure in regions where the cloud composition is homogeneous (Figs. 10, 11).

The results particularly highlight the importance of submillimeter observations for combined retrievals of frozen hydrometeors. While observations at currently available microwave frequencies provide information complementary to that from a radar only for thick clouds with very large particles $\left(D_{\mathrm{m}}>800 \mu \mathrm{m}\right.$; IWC $\left.>10^{-4} \mathrm{~kg} \mathrm{~m}^{-3}\right)$, frequencies above $200 \mathrm{GHz}$ provide additional information on cloud microphysics (Fig. 5) at smaller particles sizes and water content $\left(D_{\mathrm{m}}>200 \mu \mathrm{m}\right.$; IWC $\left.>10^{-5} \mathrm{~kg} \mathrm{~m}^{-3}\right)$.

Regarding the representation of hydrometeors in the retrieval, our results indicate that complex mixes of hydrometeors can be accurately represented using a single, suitable habit mix. In particular, our results indicate that a suitable habit should have scattering properties that are intermediate between strongly rimed and more snowflake-like particles (Figs. 4, 9).
A direct application of the synergistic retrieval algorithm developed in this study is flight campaigns involving the International Sub-millimetre Airborne Radiometer (ISMAR; Fox et al., 2017) combined for example with a radar on another aircraft or the Microwave Radar/radiometer for Arctic Clouds (MiRAC; Mech et al., 2019). The ability of the combined retrieval to constrain two moments of the PSD of frozen hydrometeors should make it a valuable tool for validating the representation of clouds in cloud-resolving or large-eddy simulations, which typically employ two-moment schemes. Moreover, since our results also indicate retrieval skill for CLWC in mixed-phase clouds, such observations can be used to study the properties of these clouds, which play an important role for the climate of the Arctic. The sensitivity to CLWC of the passive observations is also a promising indication for combined ICI and MWI retrievals.

Ultimately, spaceborne combined radar and submillimeter observations can reduce the large uncertainties in the observational record of ice hydrometeors. The Metop program provides an opportunity for a synergistic radar mission involving the MWI and ICI radiometers. Alternatively, the combination could also be realized by a dedicated small mission, such as the Earth's NexT-generation ICE mission (ENTICE) described in Jiang et al. (2019). The results presented here clearly show the potential of this approach and can provide a first step towards the development of a retrieval algorithm for a space-borne configuration. This, however, will require extending the algorithm to the more complex space-borne viewing geometry. Moreover, to quantify the potential benefits of such a mission, additional studies will be required to analyze the error sources which affect spaceborne observations. 


\section{Appendix A: Results from second test scene}

The retrieved IWC obtained using the Large Plate Aggregate for the second scene is shown in Fig. A1. Just as the first scene, this test scene contains a region in the south where the final OEM cost, shown in panel (a), is increased for the passive-only and combined retrievals. This is again a region of very dense cloud consisting of graupel and snow. Qualitatively, the results of the IWC retrieval are very similar to those from the first scene. While the passive-only retrieval provides only very low vertical resolution, both the radaronly and combined retrieval reproduce the vertical structure of the cloud well. The radar-only retrieval consistently overestimates the IWC in the scene, which is not the case for the combined retrieval.
Scatter plots for the retrieval results from the second scene are shown in Fig. A2. Except for the lack of cloud ice in the scene, the results are similar to what has been observed in the first scene: the radar-only retrieval exhibits the same systematic error for the retrieval of snow as in the first scene. Again, this is corrected by the combined retrieval for most of the tested particle shapes. The exceptions are the GEM Cloud Ice and the Large Column Aggregate particles for which the retrieval does not perform as well. 

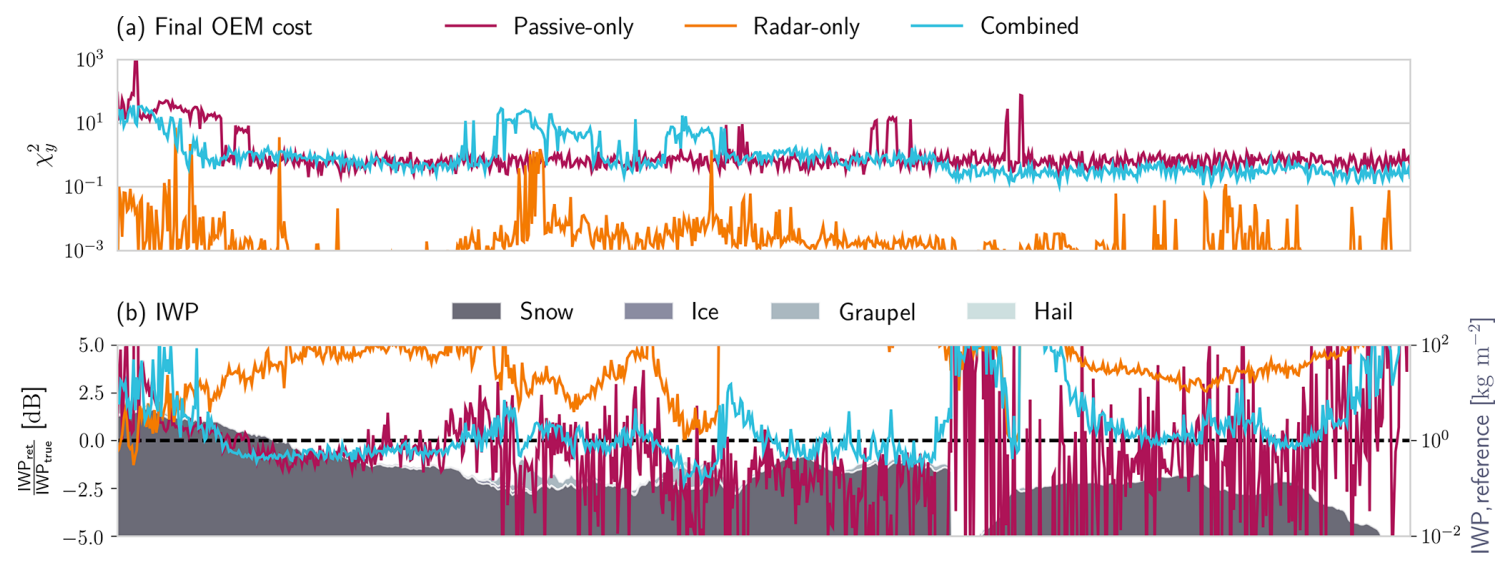

(c) Reference

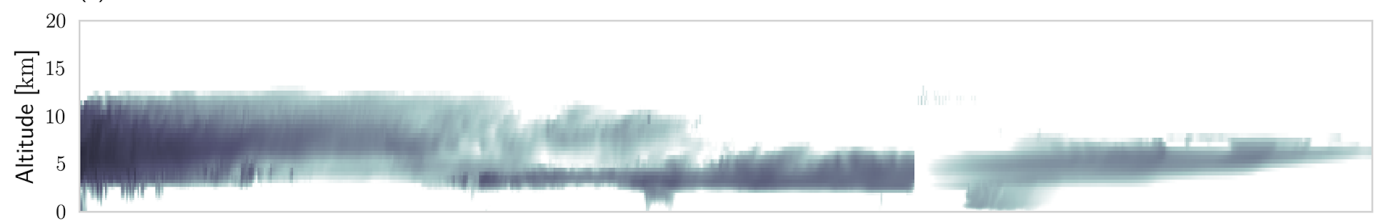

(d) Passive-only

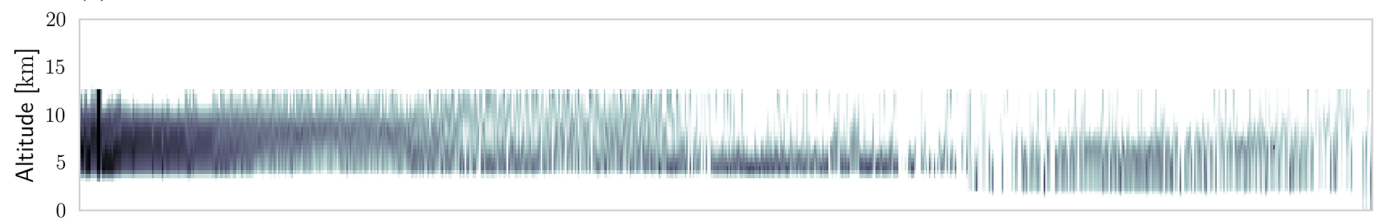

(e) Radar-only

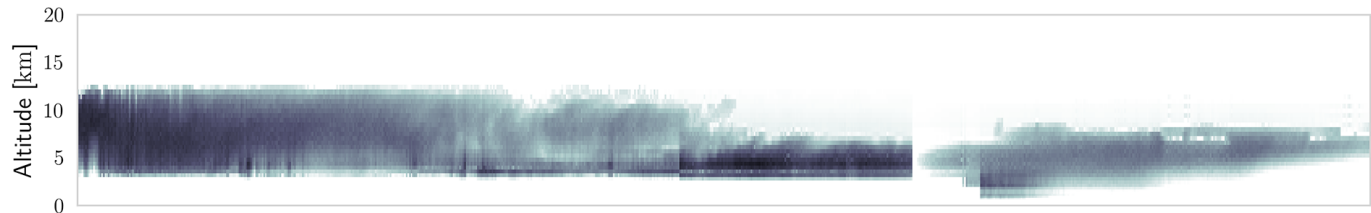

(f) Combined

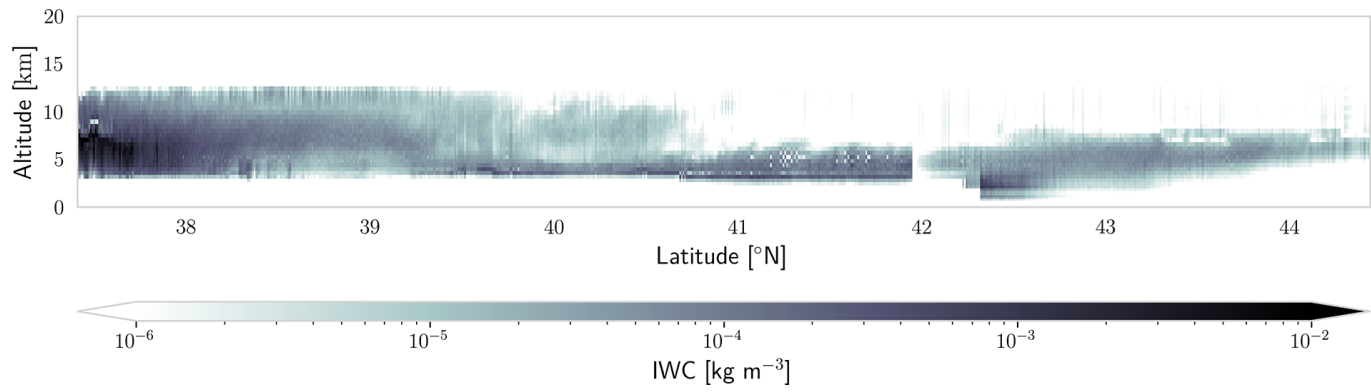

Figure A1. Results of the ice hydrometeor retrieval for the second test scene. Panel (a) displays the value of the $\chi_{y}^{2}$ diagnostic normalized by the dimension of the measurement space of the corresponding retrieval. Panel (b) shows retrieved IWP in decibels (dB) relative to the reference IWP. Reference IWP and the contributions from different hydrometeor classes are displayed by the filled areas in the background. Panel (c) displays the reference mass concentrations from the model scene. Panels (d), (e) and (f) display the retrieval results for the passiveonly, radar-only and combined retrieval, respectively. 

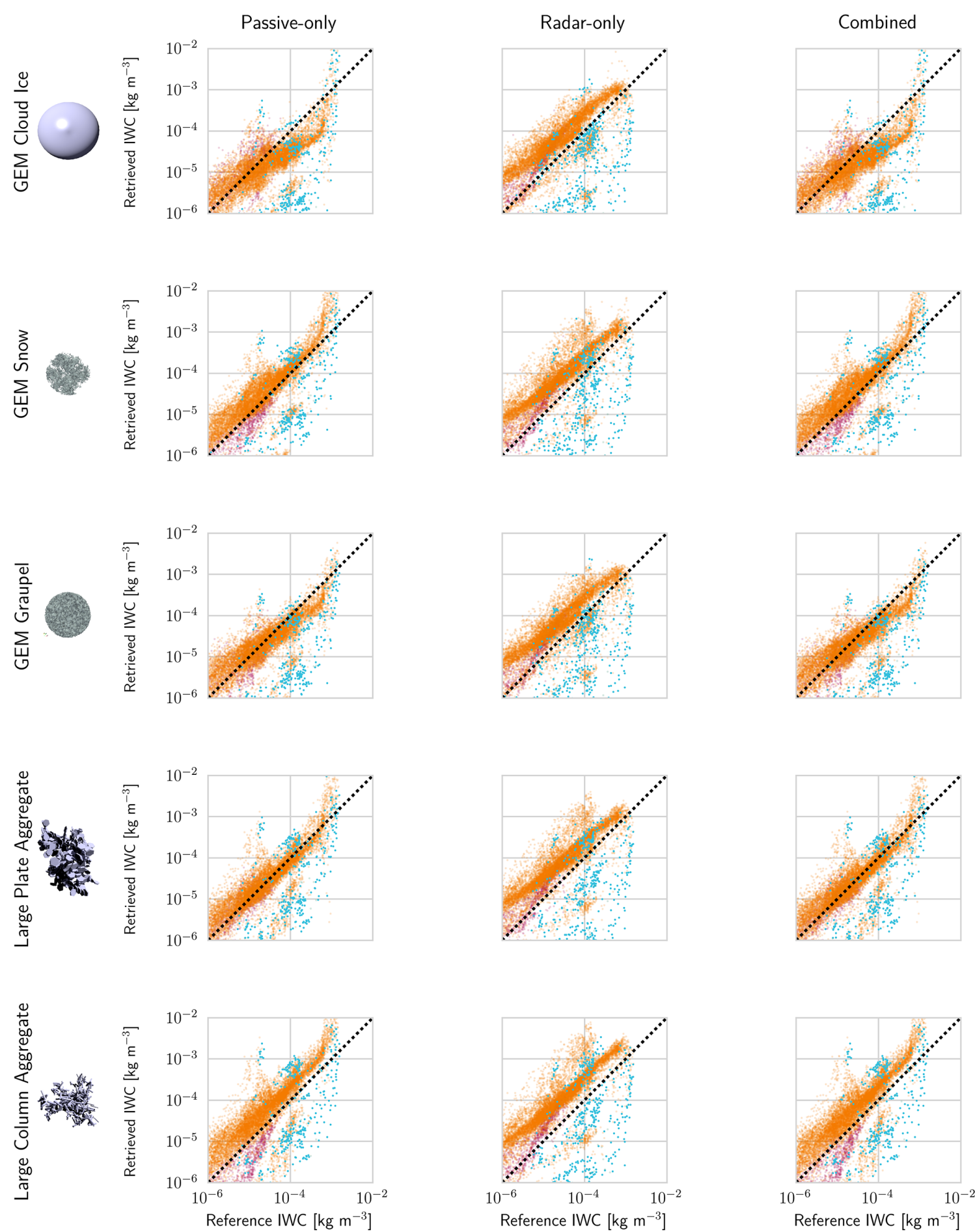

Cloud ice
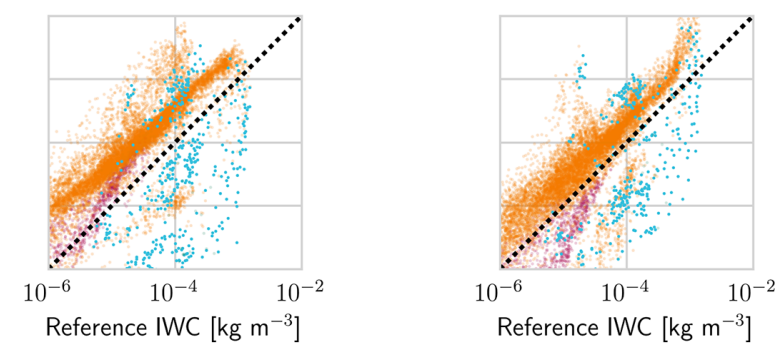

Figure A2. Scatter plots of the reference and retrieved IWC for the second test scene. The rows show the retrieval results for a given assumed ice particle model. The first column of each row displays a rendering of the particle model. The following columns display the results for the passive-only, the radar-only and the combined retrieval. 
Code availability. All code used to produce the results in this study is available from a public repository (https://doi.org/10.5281/zenodo.3467316, Pfreundschuh, 2019).

Data availability. Data to reproduce the simulations leading to the presented results will be made available on request.

Supplement. The supplement related to this article is available online at: https://doi.org/10.5194/amt-13-4219-2020-supplement.

Author contributions. SP has implemented the retrieval, performed the data analysis and written the article. PE and RL have added code to the ARTS radiative transfer model that was required to perform the presented calculations. SAB, PE, MB and SP have collaborated on the study that led to the results presented here. DD and RE have contributed to the conceptualization of the study through comments and advice.

Competing interests. The authors declare that they have no conflict of interest.

Acknowledgements. The combined and radar-only retrievals were developed as part of the ESA-funded study "Scientific Concept Study for Wide-Swath High-Resolution Cloud Profiling" (Contract number: 4000119850/17/NL/LvH). The authors would like to thank study manager Tobias Wehr for his valuable input and guidance.

Furthermore, the authors would like to acknowledge the work of Zhipeng Qu, Howard Barker and Jason Cole from Environment and Climate Change Canada who produced the model scenes that were used to test the retrieval.

Stefan A. Buehler is contributing to the Center for Earth System Research and Sustainability (CEN) of Universität Hamburg.

The computations for this study were performed using several freely available programming languages and software packages, most prominently the Python language (The Python Language Foundation, 2018), the IPython computing environment (Perez and Granger, 2007), the numpy package for numerical computing (van der Walt et al., 2011) and matplotlib for generating figures (Hunter, 2007).

The computations were performed on resources at Chalmers Centre for Computational Science and Engineering (C3SE) provided by the Swedish National Infrastructure for Computing (SNIC)

Financial support. This research has been supported by the Swedish National Space Agency (grant nos. 150/14 and 166/18).

Review statement. This paper was edited by Pavlos Kollias and reviewed by three anonymous referees.

\section{References}

Aires, F., Prigent, C., Buehler, S. A., Eriksson, P., Milz, M., and Crewell, S.: Towards more realistic hypotheses for the information content analysis of cloudy/precipitating situations - Application to a hyperspectral instrument in the microwave, Q. J. Roy. Meteor. Soc., 145, 1-14, https://doi.org/10.1002/qj.3315, 2019.

Birman, C., Mahfouf, J.-F., Milz, M., Mendrok, J., Buehler, S. A., and Brath, M.: Information content on hydrometeors from millimeter and sub-millimeter wavelengths, Tellus, 69, 1271562 , https://doi.org/10.1080/16000870.2016.1271562, 2017.

Bony, S., Stevens, B., Frierson, D. M., Jakob, C., Kageyama, M., Pincus, R., Shepherd, T. G., Sherwood, S. C., Siebesma, A. P., Sobel, A. H., Watanabe, M., and Webb, M. J.: Clouds, circulation and climate sensitivity, Nat. Geosci., 8, 261, https://doi.org/10.1038/ngeo2398, 2015.

Boucher, O., Randall, D., Artaxo, P., Bretherton, C., Feingold, G., Forster, P., Kerminen, V.-M., Kondo, Y., Liao, H., Lohmann, U., Rasch, P., Satheesh, S., Sherwood, S., Stevens, B., and Zhang, X.: Clouds and Aerosols, book section 7, Cambridge University Press, Cambridge, United Kingdom and New York, NY, USA, 571-658, https://doi.org/10.1017/CBO9781107415324.016, 2013.

Brath, M., Fox, S., Eriksson, P., Harlow, R. C., Burgdorf, M., and Buehler, S. A.: Retrieval of an ice water path over the ocean from ISMAR and MARSS millimeter and submillimeter brightness temperatures, Atmos. Meas. Tech., 11, 611-632, https://doi.org/10.5194/amt-11-611-2018, 2018.

Buehler, S. A., Mendrok, J., Eriksson, P., Perrin, A., Larsson, R., and Lemke, O.: ARTS, the Atmospheric Radiative Transfer Simulator - version 2.2, the planetary toolbox edition, Geosci. Model Dev., 11, 1537-1556, https://doi.org/10.5194/gmd-111537-2018, 2018.

Cazenave, Q., Ceccaldi, M., Delanoë, J., Pelon, J., Groß, S., and Heymsfield, A.: Evolution of DARDAR-CLOUD ice cloud retrievals: new parameters and impacts on the retrieved microphysical properties, Atmos. Meas. Tech., 12, 2819-2835, https://doi.org/10.5194/amt-12-2819-2019, 2019.

Côté, J., Gravel, S., Méthot, A., Patoine, A., Roch, M. and Staniforth, A.: The operational CMC-MRB global environmental multiscale (GEM) model. Part I: Design considerations and formulation, Mon. Weather Rev., 126, 1373-1395, https://doi.org/10.1175/15200493(1998)126<1373:TOCMGE>2.0.CO;2, 1998.

Delanoë, J., Protat, A., Testud, J., Bouniol, D., Heymsfield, A. J., Bansemer, A., Brown, P., and Forbes, R.: Statistical properties of the normalized ice particle size distribution, J. Geophys. Res.Atmos., 110, D10201, https://doi.org/10.1029/2004JD005405, 2005.

Delanoë, J., Heymsfield, A. Protat, A., Bansemer, A., and Hogan, R.: Normalized particle size distribution for remote sensing application, J. Geophys. Res.-Atmos., 119, 4204-4227, https://doi.org/10.1002/2013JD020700, 2014.

Duncan, D. I. and Eriksson, P.: An update on global atmospheric ice estimates from satellite observations and reanalyses, Atmos. Chem. Phys., 18, 11205-11219, https://doi.org/10.5194/acp-18 11205-2018, 2018.

Ekelund, R., Eriksson, P., and Pfreundschuh, S.: Using passive and active observations at microwave and sub-millimetre wave- 
lengths to constrain ice particle models, Atmos. Meas. Tech., 13, 501-520, https://doi.org/10.5194/amt-13-501-2020, 2020.

Eliasson, S., Buehler, S. A., Milz, M., Eriksson, P., and John, V. O.: Assessing observed and modelled spatial distributions of ice water path using satellite data, Atmos. Chem. Phys., 11, 375391, https://doi.org/10.5194/acp-11-375-2011, 2011.

Eriksson, P., Ekelund, R., Mendrok, J., Brath, M., Lemke, O., and Buehler, S. A.: A general database of hydrometeor single scattering properties at microwave and submillimetre wavelengths, Earth Syst. Sci. Data, 10, 1301-1326, https://doi.org/10.5194/essd-10-1301-2018, 2018.

Eriksson, P., Rydberg, B., Mattioli, V., Thoss, A., Accadia, C., Klein, U., and Buehler, S. A.: Towards an operational Ice Cloud Imager (ICI) retrieval product, Atmos. Meas. Tech., 13, 53-71, https://doi.org/10.5194/amt-13-53-2020, 2020.

Evans, K. F., Wang, J. R., Racette, P. E., Heymsfield, G., and Li, L.: Ice Cloud Retrievals and Analysis with the Compact Scanning Submillimeter Imaging Radiometer and the Cloud Radar System during CRYSTAL FACE, J. Appl. Meteorol., 44, 839-859, https://doi.org/10.1175/JAM2250.1, 2005.

Fox, S., Lee, C., Moyna, B., Philipp, M., Rule, I., Rogers, S., King, R., Oldfield, M., Rea, S., Henry, M., Wang, H., and Harlow, R. C.: ISMAR: an airborne submillimetre radiometer, Atmos. Meas. Tech., 10, 477-490, https://doi.org/10.5194/amt-10-4772017, 2017.

Fox, S., Mendrok, J., Eriksson, P., Ekelund, R., O'Shea, S. J., Bower, K. N., Baran, A. J., Harlow, R. C., and Pickering, J. C.: Airborne validation of radiative transfer modelling of ice clouds at millimetre and sub-millimetre wavelengths, Atmos. Meas. Tech., 12, 1599-1617, https://doi.org/10.5194/amt12-1599-2019, 2019.

Geer, A. J., Baordo, F., Bormann, N., Chambon, P., English, S. J., Kazumori, M., Lawrence, H., Lean, P., Lonitz, K., and Lupu, C.: The growing impact of satellite observations sensitive to humidity, cloud and precipitation, Q. J. Roy. Meteor. Soc., 143, 31893206, https://doi.org/10.1002/qj.3172, 2017.

Grecu, M., Olson, W. S., and Anagnostou, E. N.: Retrieval of Precipitation Profiles from Multiresolution, Multifrequency Active and Passive Microwave Observations, J. App. Meteorol., 43, 562-575, https://doi.org/10.1175/15200450(2004)043<0562:ROPPFM>2.0.CO;2, 2004.

Grecu, M., Olson, W. S., Munchak, S. J., Ringerud, S., Liao, L., Haddad, Z., Kelley, B. L., and McLaughlin, S. F.: The GPM Combined Algorithm, J. Atmos. Ocean. Tech., 33, 2225-2245, https://doi.org/10.1175/JTECH-D-16-0019.1, 2016.

Greenwald, T. J. and Christopher, S. A.: Effect of cold clouds on satellite measurements near $183 \mathrm{GHz}, \mathrm{J}$. Geophys. Res.-Atmos., 107, AAC 3-1-AAC 3-8, https://doi.org/10.1029/2000JD000258, 2002.

Grützun, V., Buehler, S. A., Kluft, L., Mendrok, J., Brath, M., and Eriksson, P.: All-sky information content analysis for novel passive microwave instruments in the range from 23.8 to $874.4 \mathrm{GHz}$, Atmos. Meas. Tech., 11, 4217-4237, https://doi.org/10.5194/amt-11-4217-2018, 2018.

Hou, A. Y., Kakar, R. K., Neeck, S., Azarbarzin, A. A., Kummerow, C. D., Kojima, M., Oki, R., Nakamura, K., and Iguchi, T.: The Global Precipitation Measurement Mission, B. Am. Meteorol. Soc., 95, 701-722, https://doi.org/10.1175/BAMS-D-1300164.1, 2014.
Hunter, J. D.: Matplotlib: A 2D graphics environment, Comput. Sci. Eng., 9, 90-95, https://doi.org/10.1109/MCSE.2007.55, 2007.

Jiang, J. H., Yue, Q., Su, H., Kangaslahti, P., Lebsock, M., Reising, S., Schoeberl, M., Wu, L., and Herman, R. L.: Simulation of Remote Sensing of Clouds and Humidity From Space Using a Combined Platform of Radar and Multifrequency Microwave Radiometers, Earth and Space Science, 6, 1234-1243, https://doi.org/10.1029/2019EA000580, 2019.

Jiménez, C., Buehler, S., Rydberg, B., Eriksson, P., and Evans, K.: Performance simulations for a submillimetre-wave satellite instrument to measure cloud ice, Q. J. Roy. Meteor. Soc., 133, 129_ 149, https://doi.org/10.1002/qj.134, 2007.

Kummerow, C., Barnes, W., Kozu, T., Shiue, J., and Simpson, J.: The tropical rainfall measuring mission (TRMM) sensor package, J. Atmos. Ocean. Tech., 15, 809-817, 1998.

Kummerow, C. D., Randel, D. L., Kulie, M., Wang, N.-Y., Ferraro, R., Munchak, J. S., and Petkovic, V.: The Evolution of the Goddard Profiling Algorithm to a Fully Parametric Scheme, J. Atmos. Ocean. Tech., 32, 2265-2280, https://doi.org/10.1175/JTECH-D-15-0039.1, 2015.

Liebe, H. J., Hufford, G. A., and Cotton, M. G.: Propagation modeling of moist air and suspended water/ice particles at frequencies below $1000 \mathrm{GHz}$, in: AGARD conference proceedings 542: Atmospheric propagation effects through natural and man-made obscurants for visible to mm-wave radiation, Palma de Mallorca, Spain, 17-20 May 1993, 3.1-3.10, 1993.

Mech, M., Kliesch, L.-L., Anhäuser, A., Rose, T., Kollias, P., and Crewell, S.: Microwave Radar/radiometer for Arctic Clouds (MiRAC): first insights from the ACLOUD campaign, Atmos. Meas. Tech., 12, 5019-5037, https://doi.org/10.5194/amt-125019-2019, 2019.

Milbrandt, J. A. and Yau, M. K.: A multimoment bulk microphysics parameterization. Part II: A proposed three-moment closure and scheme description, J. Atmos. Sci., 62, 3065-3081, https://doi.org/10.1175/JAS3534.1, 2005.

Munchak, S. J. and Kummerow, C. D.: A Modular Optimal Estimation Method for Combined Radar-Radiometer Precipitation Profiling, J. Atmos. Ocean. Tech., 50, 433-448, https://doi.org/10.1175/2010JAMC2535.1, 2011.

Perez, F. and Granger, B. E.: IPython: A System for Interactive Scientific Computing, Comput. Sci. Eng., 9, 21-29, https://doi.org/10.1109/MCSE.2007.53, 2007.

Pfreundschuh, S.: Microwave Clour Retrieval Framework (MCRF), Zenodo, https://doi.org/10.5281/zenodo.3467316, 2019.

Pfreundschuh, S., Eriksson, P., Duncan, D., Rydberg, B., Håkansson, N., and Thoss, A.: A neural network approach to estimating a posteriori distributions of Bayesian retrieval problems, Atmos. Meas. Tech., 11, 4627-4643, https://doi.org/10.5194/amt11-4627-2018, 2018.

Prigent, C., Aires, F., Wang, D., Fox, S., and Harlow, C.: Sea-surface emissivity parametrization from microwaves to millimetre waves, Q. J. R. Meteorol. Soc., 143, 596-605, https://doi.org/10.1002/qj.2953, 2017.

Rodgers, C. D.: Inverse methods for atmospheric sounding: theory and practice, World scientific, vol. 2, https://doi.org/10.1142/3171, 2000.

Rosenkranz, P. W.: Absorption of microwaves by atmospheric gases, in: Atmospheric remote sensing by microwave radiome- 
try, edited by: Janssen, M. A., 37-90, John Wiley and Sons, Inc., New York, USA, 1993.

Rosenkranz, P. W.: Water vapor microwave continuum absorption: A comparison of measurements and models, Radio Sci., 33, 919 928, https://doi.org/10.1029/98RS01182, 1998.

Stamnes, K., Tsay, S.-C., Wiscombe, W., and Laszlo, I.: DISORT, a general-purpose Fortran program for discrete-ordinate-method radiative transfer in scattering and emitting layered media: documentation of methodology, Tech. rep., Dept. of Physics and Engineering Physics, Stevens Institute of Technology, Hoboken, New Jersey, USA, 2000.

Stephens, G. L., Vane, D. G., Boain, R. J., Mace, G. G., Sassen, K., Wang, Z., Illingworth, A. J., O'connor, E. J., Rossow, W. B., Durden, S. L., Miller, S. D., Austin, R. T., Benedetti, A., and Mitrescu, C. A.: THE CLOUDSAT MISSION AND THE A-TRAIN, B. Am. Meteorol. Soc., 83, 1771-1790, https://doi.org/10.1175/BAMS-83-12-1771, 2002.

Tanelli, S., Durden, S. L., Im, E., Pak, K. S., Reinke, D. G., Partain, P., Haynes, J. M., and Marchand, R. T.: CloudSat's Cloud Profiling Radar After Two Years in Orbit: Performance, Calibration, and Processing, IEEE T. Geosci. Remote, 46, 3560-3573, https://doi.org/10.1109/TGRS.2008.2002030, 2008.

The Python Language Foundation: The Python Language Reference, https://docs.python.org/3/reference/index.html (last access: 10 August 2020), 2018. van der Walt, S., Colbert, S. C., and Varoquaux, G.: The NumPy Array: A Structure for Efficient Numerical Computation, Computing in Science Engineering, 13, 22-30, https://doi.org/10.1109/MCSE.2011.37, 2011.

Waliser, D. E., Li, J.-L. F., Woods, C. P., Austin, R. T., Bacmeister, J., Chern, J., Del Genio, A., Jiang, J. H., Kuang, Z., Meng, H., Minnis, P., Platnick, S., Rossow, W. B., Stephens, G. L., SunMack, S., Tao, W.-K., Tompkins, A. M., Vane, D. G., Walker, C., and Wu, D.: Cloud ice: A climate model challenge with signs and expectations of progress, J. Geophys. Res.-Atmos., 114, D00A21, https://doi.org/10.1029/2008JD010015, 2009.

Wang, D., Prigent, C., Aires, F., and Jimenez, C.: A Statistical Retrieval of Cloud Parameters for the Millimeter Wave Ice Cloud Imager on Board MetOp-SG, IEEE Access, 5, 4057-4076, https://doi.org/10.1109/ACCESS.2016.2625742, 2017.

Xie, X., Crewell, S., Löhnert, U., Simmer, C., and Miao, J.: Polarization signatures and brightness temperatures caused by horizontally oriented snow particles at microwave bands: Effects of atmospheric absorption, J. Geophys. Res.-Atmos., 120, 6145$6160,2015$. 